DAMTP-95-07

hep-th/9502106

\title{
Multi-boundary effects in Dirichlet String Theory
}

\author{
Michael Gutperle日 \\ D.A.M.T.P., Silver Street \\ Cambridge, CB3 9EW, U. K.
}

\begin{abstract}
String amplitudes with an arbitrary number of world-sheet boundaries on which the coordinates satisfy Dirichlet boundary conditions are analyzed in a path integral framework. Special attention is payed to the novel divergences associated with such conditions. Certain helicity amplitudes involving massless closed-string states are free of such divergences to all orders in perturbation theory and their behavior can be analysed unambiguously. The high energy fixed-angle behavior of these amplitudes is discussed in the presence of one or two boundaries and the asymptotic behavior of the amplitudes is shown to be power behaved.
\end{abstract}

\section{Introduction}

In open string theories one has to impose boundary conditions on the fields living on the world sheet in order to canonically quantize the theory or to define a path integral. For the usual bosonic open string one imposes Neumann

\footnotetext{
${ }^{1}$ e-mail: M.Gutperle@amtp.cam.ac.uk, on leave at TH-Division, CERN, Geneva
} 
conditions on the space-time coordinates $X^{\mu}$, which means that the normal derivatives of $X^{\mu}$ are set equal to zero at the boundary. Another possibility consistent with conformal invariance [1] is to impose constant (Dirichlet) boundary conditions. This means that each boundary is mapped into a single spacetime point.

This idea was first employed to formulate off-shell extensions of dual amplitudes by coupling dual models to external currents, for open strings [2], [3] and for closed strings [4]. See also [5] for a path integral approach to off-shell amplitudes using Dirichlet boundary conditions. The theory with Dirichlet boundaries is formally related to the Neumann theory by target space duality of the compactified theory [6], [7], but this will not be considered here since we will be concerned with flat Minkowski space as a target space manifold.

This paper will investigate the theory with Dirichlet boundaries in which the boundary positions are integrated over space-time [6]. This ensures that no momentum can enter or leave through any boundary leading to a modification of the closed string theory. The insertion of a single Dirichlet boundary was considered in [6] and the case of two boundary insertions was considered in [8], 9]. The theory has drastically altered fixed angle scattering amplitudes for closed string states which exhibit pointlike behavior in the high energy fixed angle regime. This paper will discuss properties of the string perturbation expansion for such a theory on oriented Riemann surfaces although the doubling procedure that we use can also be applied to unoriented surfaces and many features carry over to Dirichlet boundary insertions on nonoriented Riemann surfaces.

The organization of the paper is as follows: In section 2 we describe the Dirichlet open string by doubling the bordered world sheet and all the quantities which are necessary for the evaluation of the open string path integral are expressed in terms of quantities on the compact double. The path integral with an arbitrary number of world-sheet boundaries on which the coordinates satisfy Dirichlet boundary conditions is performed and different applications for the result are given. Certain new kinds of divergences arise in the Dirichlet theory that originate from the two lowest states $(N=0, N=1)$ in the open string Hilbert space. A general analysis of these divergences and of the degenerations of the world sheet where they occur is given. In section 3 and 1 we analyze the scattering amplitudes with one and two Dirichlet boundary insertions. The analysis of the divergences of section 2 is illustrated 
in these two cases. We focus on high energy fixed angle scattering and show that for certain helicity amplitudes of massless tensor states the behavior of the amplitudes can be analyzed unambiguously. The scattering behavior is disentangled from the divergences which arise at the boundary of moduli space and it is shown that the amplitudes are power behaved with respect to the center of mass energy.

In appendix A a modification of the Green function is discussed which arises in the functional integration. The rôle of the $N=1$ state and its interpretation as a Lagrange multiplier field will be described in Appendix B. In a scheme due to Polchinski [10] the combinatorics of the boundary insertions are altered and this leads to a cancellation of the divergence caused by this field. This mechanism is briefly illustrated in Appendix C.

\section{General Considerations}

The action of the bosonic string in flat space time is given by

$$
S=\frac{1}{4 \pi \alpha^{\prime}} \int_{\Sigma} d^{2} \sigma \sqrt{\operatorname{det} g} g^{a b} \partial_{a} X^{\mu} \partial_{b} X_{\mu}
$$

Scattering amplitudes for closed bosonic strings are defined perturbatively by the Polyakov-path-integral over surfaces of different topologies and given by expectation values of vertex-operators inserted on the world sheet

$$
\Gamma=\sum_{\text {topologies }} \int \frac{d g d X}{\operatorname{vol}(\operatorname{diff} \triangleleft w e y l)} \exp (-S(X, g)) \prod_{i=1}^{n} \int d^{2} \sigma_{i} V\left(k_{i}, X\left(\sigma_{i}\right)\right)
$$

Holomorphic factorization [11] of the path integral measure implies that the vacuum amplitude with no vertex operator insertions can be written as an integral over the complex moduli space and the integrand is given by a modular form on this space

$$
Z_{g}=\int_{\text {moduli }} \prod_{k=1}^{3 g-3} d^{2} m_{k}\left|\sigma\left(m_{1}, \cdots, m_{k}\right)\right|^{2} \operatorname{det}(\operatorname{Im}(\tau))^{-13}
$$

Here $\prod_{k=1}^{3 g-3} d^{2} m_{k}\left|\sigma\left(m_{1}, \cdots, m_{k}\right)\right|^{2}$ is due to the evaluation of the path integral and contains the determinants of scalar and vector laplacian of the Riemann surface, $\sigma(m)$ is a modular form of weight -13 on the moduli space of complex 
dimension $3 g-3$. It is parameterized by the coordinates $m_{i}$ which correspond to the deformations of the complex structure of the surface. The measure $\sigma(m)$ can be expressed in terms of well known functionals which are defined on the Riemann surface corresponding to the moduli $m$ [12]. The measure can also be represented by a path integral over ghosts in the usual way and one gets

$$
Z_{g}=\int_{\text {moduli }} \prod_{k=1}^{3 g-3} d^{2} m_{k} \int D X D b D c \prod_{i=1}^{3 g-3}\left|\left(\mu_{i}, b\right)\right|^{2} e^{-S_{g h}-S_{X}}
$$

Here the $\left(\mu_{i}, b\right)$ is the pairing of a Beltrami differential corresponding to the modulus $m$ with a b-ghost to absorb the zero modes of the b-ghost. This formula is valid for $g>1$, for low genus one also has to take care of the zero modes for the c-ghosts corresponding to conformal Killing vectors.

On a compact Riemann surface we can define an intersection pairing $J$ and a canonical homology basis $\left(a_{i}, b_{i}\right), i=1, \cdots, g$ which satisfies

$$
J\left(a_{i}, a_{j}\right)=J\left(b_{i}, b_{j}\right)=0 ; J\left(a_{i}, b_{j}\right)=-J\left(b_{j}, a_{i}\right)=\delta_{i j}
$$

Together with the homology cycles come the Abelian differentials of the first kind $\omega_{i}$, which define the period matrix $\tau$ by

$$
\oint_{a_{i}} \omega_{j}=\delta_{i j} ; \oint_{b_{i}} \omega_{j}=\tau_{i j}
$$

\subsection{Doubling of a bordered surface}

In mathematics [13], conformal field theory [1] and open string theory one encounters Riemann surfaces which have boundaries, and therefore are not compact. A standard way of dealing with this is to represent the bordered Riemann surface as a quotient of a compact Riemann surface under an antiholomorphic involution. This is called doubling and has been used to analyze the loop amplitudes of the standard (Neumann) open string theories [14], [15]. Using this the well known results for closed strings can also be applied in the context of Dirichlet boundary conditions.

Starting with a (oriented) surface $\Sigma$ with $b$ boundaries $\partial \Sigma_{i}, i=1, \cdots, b$ and $h$ handles, we take a topological copy of $\Sigma$ and glue the two surfaces together by an orientation reversing involution $I$ such that $\Sigma=\bar{\Sigma} / I$ and 
$I\left(\partial \Sigma_{i}\right)=\partial \Sigma_{i}$ (See figure 1). A complex structure on $\bar{\Sigma}$ can be defined from one on $\Sigma$ by applying the Schwartz reflection principle : For a local complex coordinate $z(p)$ near $p \epsilon \Sigma$ a coordinate at $I(p)$ is given via $z(I(p))=\bar{z}(p)$. This makes $\bar{\Sigma}$ into a complex manifold and the involution $I$ anticonformal. The double is a compact surface of genus $g=2 h+b-1$. In general the involution $I$ acts nontrivially on the homology cycles $a_{i}, b_{i} ; 1, \cdots, g$

$$
\begin{aligned}
I\left(a_{i}\right) & =\sum_{j} C_{i j} a_{j} \\
I\left(b_{i}\right) & =\sum_{j} D_{i j} a_{j}-\sum_{j} C_{i j} b_{j}
\end{aligned}
$$

With certain constraints on the matrices $C, D$ coming from the invariance of (5) under $I$. A convenient choice of the homology cycles which is possible [16] is given by $D_{i j}=\delta_{i j}$. In the most general cases one cannot set $C_{i j}=0$, but when there are no handles, i.e. $h=0$, this can be done. The invariance of the a-cycles means that they either lie on the boundary or are sums of cycles which get interchanged by $I$. An important term which appears in the expressions of the functional determinants is

$$
R_{\Sigma}=\operatorname{det}\left\{\frac{1}{2}(1-D) \operatorname{Im}(\tau)^{-1}+\frac{1}{2}(1+D) \operatorname{Im}(\tau)\right\}
$$

With the special choice above this gives $R_{\Sigma}=\operatorname{Im}(\tau)^{-1}$. In [15] the functional determinants which occur during the reduction of the path integral to an integral over moduli space for a bordered surface were obtained from the determinants for the compact double. The measure on moduli space of the open string is given by

$$
\prod_{k=1}^{3 g-3} d m_{k}\left|\sigma\left(m_{1}, \cdots, m_{k}\right)\right|[\operatorname{det} \operatorname{Im}(\tau)]^{-13 / 2} R_{\Sigma}^{+}{ }^{-13 / 2}
$$

In principle this is the 'holomorphic square root' of (3) and is to be integrated over a real slice of the moduli space of the genus g surface. The involution $I$ also acts on the moduli space and in essence the part of moduli space which is invariant under $I$ (those deformations which deform both $\Sigma$ and $I(\Sigma)$ symmetrically) is integrated over. The sign in (10) is determined by the boundary condition on the spacetime fields $X$, plus for Neumann and minus for Dirichlet boundary conditions, see [15] for details. 


\subsection{Gaussian integration}

For a surface with a boundary the integration by parts in the action (11) gives a boundary term contribution, we drop the antiholomorphic arguments for notational convenience. The most general vertex operator has the form $V(k, \zeta)=: A(\partial X, . ., \zeta) e^{i k X}:$, where A symbolically indicates the dependence on the polarization tensors $\zeta$ and the derivatives of $X$ and the ellipses denote normal ordering. The functional integral with a product of such vertex operators can be evaluated by exponentiating the prefactors $A$. This leads to terms involving the derivatives of the Green function but without any momentum dependence. Since we focus on the momentum dependence of the amplitudes leaving the prefactors unexponentiated and combining the universal factors $\exp (i k X)$ gives the exponential part of the integrand $\epsilon$

$$
\epsilon=-\frac{1}{4 \pi \alpha^{\prime}} \int_{\Sigma} X \partial \bar{\partial} X+\frac{1}{4 \pi \alpha^{\prime}} \int_{\partial \Sigma} X \partial X+i \sum_{i=1}^{n} k_{i}^{\mu} X_{\mu}\left(z_{i}\right)
$$

To integrate out the $X$ in the path integral (2) $X$ is separated into a classical part and a quantum fluctuation $\xi(z)$

$$
X^{\mu}(z)=\xi^{\mu}(z)+2 \pi i \alpha^{\prime} \sum_{i=1}^{n} k_{i}^{\mu} G^{D}\left(z, z_{i}\right)+X_{c l}^{\mu}(z)
$$

$G^{D}(z, w)$ is the (uniquely defined) Dirichlet Green function on the surface $\Sigma$ with $b$ boundary components $\partial \Sigma_{i}, i=1, \cdots, b$ which vanishes on all boundary components. The quantum fluctuation $\xi$ is also chosen to vanish at the boundaries. The classical solution $X_{c l}(z)$ satisfies the Laplace-equation in $\Sigma$ and satisfies on the boundary $\left.X_{c l}(z)\right|_{z \epsilon \partial \Sigma_{i}}=Y_{i}$. It is easy to see that $X_{c l}$ can be expressed implicitly with the help of the Green function in the following way

$$
X_{c l}(z)=2 i \sum_{i=1}^{b} \oint_{\partial \Sigma_{i}} d w X_{c l}(w) \partial_{w} G^{D}(z, w)
$$

The first $b$ a-cycles are chosen to lie on the boundary and the boundaries are represented by

$$
\partial \Sigma_{1}=a_{1}, \partial \Sigma_{2}=a_{2}-a_{1}, \cdots, \partial \Sigma_{b-1}=a_{b-1}-a_{b-2}, \partial \Sigma_{b}=\partial \Sigma-a_{b-1}
$$


With this definition the $X_{c l}$ can be expressed as an integral over the a-cycles and the boundary values $Y_{i}$

$$
X_{c l}(z)=2 i \sum_{i=1}^{b-1}\left(Y_{i}-Y_{i+1}\right) \oint_{a_{i}} d w \partial_{w} G^{D}(z, w)+Y_{b}
$$

The Green function on the Riemann surface $\Sigma G_{\Sigma}^{D}(z, w)$ can be given in terms of the Green function on the double $\bar{\Sigma}$ which in turn can be expressed in terms of the prime-form $E$ and abelian differentials of the first kind $\omega_{i}$ [17]

$$
\begin{gathered}
G_{\Sigma}^{D}(z, w)=G_{\bar{\Sigma}}(z, w)-G_{\bar{\Sigma}}(z, I(w)) \\
G_{\bar{\Sigma}}=-\frac{1}{4 \pi} \ln |E(z, w)|^{2}+\frac{1}{2} \operatorname{Im} \int_{z}^{w} \omega_{i} \operatorname{Im} \tau_{i j}^{-1} \operatorname{Im} \int_{z}^{w} \omega_{j}+F(z, \bar{z})+F(w, \bar{w})
\end{gathered}
$$

The Gaussian integration can be done by inserting (12) into (11). Omitting a term quadratic in the quantum fluctuations $\xi$, which gives the determinant of the Laplacian upon functional integration of $\xi$, the classical contribution to the exponent $\epsilon_{c l}$ is

$$
\epsilon_{c l}=-\alpha^{\prime} \pi \sum_{i j} k_{i} k_{j} G_{\Sigma}^{D}\left(z_{i}, z_{j}\right)+\frac{1}{4 \pi \alpha^{\prime}} \int_{\partial \Sigma} X_{c l} \partial X_{c l}+i \sum_{i} k_{i} X_{c l}\left(z_{i}\right)
$$

Using (13) and (17) the double derivative of the Green function is given by

$$
\partial_{z} \partial_{w} G^{D}(z, w)=-1 /(4 \pi) \omega(z, w)-1 / 2 \sum_{i, j} \omega_{i}(z) \operatorname{Im} \tau_{i j}^{-1} \omega_{j}(w)
$$

Here $\omega(z, w)$ is the abelian differential of the third kind which vanishes when integrated along a-cycles. The second term on the r.h.s. of (18) can then be evaluated with the help of (6).

$$
\begin{aligned}
\int_{\partial \Sigma} X_{c l} \partial X_{c l} & =+2 i \sum_{i j}\left(Y_{i}-Y_{i+1}\right)\left(Y_{j}-Y_{j+1}\right) \oint_{a_{i}} \oint_{a_{j}} \partial_{z} \partial_{w} G^{D}(z, w) \\
& =-i \sum_{i, j}\left(Y_{i}-Y_{i+1}\right) I m \tau_{i j}^{-1}\left(Y_{j}-Y_{j+1}\right)
\end{aligned}
$$

This term and the third term on the r.h.s. of (18) give the explicit dependence of the amplitude on the boundary values $Y_{i}$. There are several uses for these formulas: 
- If there are no vertex operator insertions (20) is the only contribution in $\epsilon_{c l}$ from the Gaussian integration. We can interpret these amplitudes which depend on $Y_{i}-Y_{i+1}$ as space time correlation functions. These functions have interesting singularity structure with singularities outside, on and inside the lightcone [6]. Putting together (10) and (20) gives with $\Delta Y_{i}=Y_{i}-Y_{i+1}$ for the special case of no handles, i.e. $h=0$

$$
A(\Delta Y)=\int \prod_{k=1}^{3 g-3} d m_{k}|\sigma(m)| \operatorname{det} \operatorname{Im}(\tau)^{-13} \exp \left(\frac{1}{2 \pi \alpha^{\prime}} \sum_{i, j} \Delta Y_{i} \operatorname{Im}\left(\tau_{i j}\right)^{-1} \Delta Y_{j}\right)
$$

Note that the $\Delta Y_{i}$ enter this expression just like the loop momenta enter the expression for the partition function in the Neumann theory. Integrating over the $\Delta Y_{i}$ gives

$$
\int \prod_{i=1}^{b-1} d^{26} \Delta Y_{i} A(\Delta Y)=\left(2 \pi \alpha^{\prime}\right)^{13} \int \prod_{k=1}^{3 g-3} d m_{k}|\sigma(m)|
$$

This is the same expression as the Neumann partition function given by (10) identifying the loop momenta $P_{i}$ with $\Delta Y_{i} /\left(\sqrt{2 \pi \alpha^{\prime}}\right)$

- Inserting closed string vertex operators gives amplitudes which have been interpreted as coupling of currents to the (hadronic) string [18]. One obvious thing to consider is a Fourier transformation with respect to the boundary positions. This gives off-shell amplitudes [18], [5]. Starting from (18) it is easy to see that $\epsilon_{c l}$ only depends on the $\Delta Y_{i}$ and $Y_{b}$. Defining momenta $q_{i}$ conjugate to $Y_{i}$ an overall momentum conserving delta function $\delta\left(\sum_{i} q_{i}+\sum_{j} k_{j}\right)$ is obtained from the integration of $Y_{b}$ and an integrand which then only depends on the $\Delta Y_{i}$. With new momenta $\hat{q}_{i}$ conjugate to the $\Delta Y_{i}$

$$
\begin{gathered}
A\left(k_{i}, \hat{q}_{j}\right)=\int \prod_{k=1}^{3 g-3} d m_{k}|\sigma(m)| \operatorname{det} \operatorname{Im}(\tau)^{-13} \int \prod_{k=1}^{b-1} d^{26} \Delta Y_{k} \exp \left(i \sum_{k=1}^{b-1} \hat{q}_{k} \Delta Y_{k}+\epsilon_{c l}\right) \\
=\int \prod_{k=1}^{3 g-3} d m_{k}|\sigma(m)| \exp \left\{-2 \pi \alpha^{\prime} \sum_{l, m=1}^{b-1}(b-i \hat{q})_{l} \operatorname{Im}(\tau)_{l m}(b-i \hat{q})_{m}\right\}
\end{gathered}
$$

Where $b_{j}=\sum_{i} k_{i} \oint_{a_{j}} \partial_{w} G^{D}\left(z_{i}, w\right)$. Note that the Fourier transformation has changed the powers of $\operatorname{det}(\operatorname{Im}(\tau))$ in the measure. The $\hat{q}_{i}$ are connected to the momentum of the Dirichlet boundaries and one interesting thing to 
consider is the 'deep inelastic' regime where $\left|q_{i}\right| \rightarrow \infty$ corresponding to a large momentum transfer through the boundaries into the diagram.

- Another interesting consideration is the situation when all the $Y_{i}$ are integrated independently over space time with unit weight, so all $\hat{q}_{i}$ are set to zero. This means that no momentum is allowed to flow through the boundaries. Setting $\hat{q}_{i}=0$ in (23) produces a modification of the Green function since $\epsilon_{c l}=\pi \alpha^{\prime} \sum k_{i} k_{j} \hat{G}\left(z_{i}, z_{j}\right)$ with

$$
\hat{G}^{D}(z, w)=G^{D}\left(z_{i}, z_{j}\right)-2 \sum_{l, m} \oint_{a_{l}} \partial w G^{D}\left(z_{i}, w\right) \operatorname{Im}(\tau)_{l m} \oint_{a_{m}} \partial_{w^{\prime}} G^{D}\left(z_{j}, w^{\prime}\right)
$$

In appendix $\mathrm{A}$ it is shown that the second term on the right hand side of (24) cancels the second term on the right hand side of (17) and the modified Green function is given by

$$
\hat{G}^{D}\left(z_{i}, z_{j}\right)=-\frac{1}{4 \pi}\left\{\ln \left|E\left(z_{i}, z_{j}\right)\right|^{2}-\ln \left|E\left(z_{i}, I\left(z_{j}\right)\right)\right|^{2}\right\}
$$

The resulting modified closed string theory has very different short distance properties compared to ordinary closed string theory. For example the high temperature properties [19] of this theory are similar to a QCD like theory [20]. Another probe of the short distance properties of a theory is high energy fixed angle scattering. For two particle scattering amplitudes this is the limit $s \rightarrow \infty$ while $s / t$ is fixed (s,t are Mandelstam variables defined by $\left.s=-\left(k_{1}+k_{2}\right)^{2}, t=-\left(k_{1}+k_{3}\right)^{2}\right)$. In general one considers $\left|k_{i} k_{j}\right| \rightarrow \infty$ and $\left|k_{i} k_{j}\right| /\left|k_{k} k_{l}\right|$ fixed. The absence of any short distance structure in conventional closed string theories is reflected by the exponential decrease of fixed angle scattering as a function of $s$ which is the center of mass energy. This was already noted in the early days of string theory [21, 222]. In [23] it was shown that the amplitude in this limit is dominated by a saddle point configuration and the high energy fixed angle behavior is universal to all orders in the topological expansion of perturbative string theory. Here the situation is different [6], since there is a power law fall off in $s$, which can be interpreted as pointlike scattering behavior induced by the Dirichlet boundary insertion compared to the complete lack of pointlike structure in the ordinary closed string. Because of the structure of the Green function in (16) we can represent a configuration with $n$ Vertex operators $V_{i}\left(z_{i}\right)$ with momentum $k_{i}$ by $2 n$ 
Vertex operators where $V_{n+i}$ is located at $I\left(z_{i}\right)$, which is the 'mirror image' of $z_{i}$, and has momentum $-k_{i}$. Then $\epsilon_{c l}$ clearly vanishes when all $z_{i}=I\left(z_{i}\right)$, i.e. when all the $z_{i}$ are on the boundary. In an electrostatic picture the $z_{i}$ and $I\left(z_{i}\right)$ are the positions of charges on the surface with strength $k_{i}^{\mu}$ and $-k_{i}^{\mu}$. It is clear that in the configuration above the charges annihilate each other and the energy $\epsilon_{c l}$ vanishes. The asymptotics are dominated by an endpoint integration. This means that the integration over the deviations $\delta z_{i}$ gives powers

of of the kinematical invariants. As explained later we expect the $G^{D}$ to be quadratic in the deviations and the generic behavior should be $s^{-n / 2}$ where $n$ is the number of vertices. This naive picture is complicated by the fact that the dominant region of the integrand lies on the boundary of moduli space, where we encounter new kinds of divergences. In the following sections the relevant divergences of the Dirichlet string are discussed and they are dealt with in the easiest cases.

- A different version of Dirichlet string theory was proposed by Polchinski [0]. This is defined by considering a gas of 'D-instantons'. A single Dinstanton is defined at a point $Y_{\mu}$ in space time, and is build up by an arbitrary number of disconnected (in the topological sense) world sheets, which all have the boundaries mapped to $Y$. The disconnected world sheets are really connected in space time via the common point Y. We can easily adapt our formalism to this description and it means that for each connected world sheet one has to set $\Delta Y_{i}=0$, for all $i$ and $Y_{b}=Y$. So that in (24) the second term on the r.h.s. is not present. The full theory is then given by summing over an arbitrary number of such D-instantons and integrating over their space time positions.

\subsection{Divergences in Dirichlet amplitudes}

It is well known that infinities in string amplitudes arise from the boundary of moduli space. In closed string theory these boundaries originate either when vertex operators approach each other on the world sheet or when surfaces degenerate and a cycle is pinched. In the plumbing fixture construction one represents this as a cylinder glued into the surface which becomes infinitely long [17]. The standard divergences that arise in closed bosonic string theory will not be discussed here since we are interested in new features that are due to the presence of Dirichlet boundaries. 
In open string theory there are four other types of degenerations (see figure 2): (a) vertex operator positions approaching the boundaries, (b) the separation of the surface into two disconnected parts, (c) two boundary components touching, (d) a boundary shrinking to a point. (d) corresponds to a closed string tadpole coupling to the disk, a phenomenon which is well discussed in the literature for Neumann strings [24]. We do not discuss the case (d) in any detail here since our main focus is on the novel open string divergences in the theory. The degenerations (a) to (c) can be represented in a conformal frame where a strip glued to the boundaries of the surfaces becomes infinitely long (see figure 3). This degenerating strip can be represented by vertex operator insertions for all open string states at the two points where the strip leaves the surface and a propagator joining them. Symbolically this can be represented as

$$
\begin{aligned}
& A_{(a)}=\sum_{\Phi_{1}, \Phi_{2}}\left\langle V_{1} . . V_{n-1} A_{\Phi_{1}}\right\rangle_{\Sigma_{1}}\left\langle\Phi_{1}|\Delta| \Phi_{2}\right\rangle\left\langle V_{n} A_{\Phi_{2}}\right\rangle_{D i s k} \\
& A_{(b)}=\sum_{\Phi_{1}, \Phi_{2}}\left\langle V_{1} . . V_{n-l} A_{\Phi_{1}}\right\rangle_{\Sigma_{1}}\left\langle\Phi_{1}|\Delta| \Phi_{2}\right\rangle\left\langle V_{n-l+1} . . V_{n} A_{\Phi_{2}}\right\rangle_{\Sigma_{2}} \\
& A_{(c)}=\sum_{\Phi_{1}, \Phi_{2}}\left\langle V_{1} . . V_{n} A_{\Phi_{1}} A_{\Phi_{2}}\right\rangle_{\Sigma^{\prime}}\left\langle\Phi_{1}|\Delta| \Phi_{2}\right\rangle \\
& A_{(d)}=\sum_{\hat{\Phi}_{1}, \hat{\Phi}_{2}}\left\langle V_{1} . . V_{n} A_{\hat{\Phi}_{1}}\right\rangle_{\Sigma^{\prime}}\left\langle\hat{\Phi}_{1}|\hat{\Delta}| \hat{\Phi}_{2}\right\rangle\left\langle A_{\hat{\Phi}_{2}}\right\rangle_{D}
\end{aligned}
$$

Here $A_{\Phi_{i}}$ denotes a boundary vertex operator insertion corresponding to the state $\Phi_{i}$ in the open string Hilbert space and $\Delta$ is the open string propagator, the hatted quantities $\hat{\phi}$ and $\hat{\Delta}$ denote closed string states and propagator for case $(\mathrm{d})$. The functional integral over $\Sigma$ is denoted by $\langle. .\rangle_{\Sigma}$. In the case of Dirichlet boundaries consider a free string quantized on an infinite strip of width $\pi$ with

$$
\left.X^{\mu}(\sigma, \tau)\right|_{\sigma=0}=Y_{1}^{\mu},\left.X^{\mu}(\sigma, \tau)\right|_{\sigma=\pi}=Y_{2}^{\mu}
$$

The mode expansion of the string coordinate $X$ is then given by

$$
X^{\mu}(\sigma, \tau)=Y_{1}^{\mu}+\frac{Y_{2}^{\mu}-Y_{1}^{\mu}}{\pi} \sigma+\sqrt{2 \alpha^{\prime}} \sum_{n+-\infty}^{n=\infty} \frac{1}{n} a_{n}^{\mu} \sin n \sigma e^{i n \tau}
$$

The Virasoro generators $L_{n}$ are easily obtained from this expansion and the zero mode of the energy momentum tensor $L_{0}$ is given by

$$
L_{0}^{\left(Y_{1}-Y_{2}\right)}=\frac{\left(Y_{1}-Y_{2}\right)^{2}}{4 \pi \alpha^{\prime}}+N
$$


$\mathrm{N}$ is the open string number operator given by $N=\frac{1}{2}: \sum_{n} a_{-n}^{\mu} a_{n \mu}:$. The propagator is given by $\Delta=\left(L_{0}-1\right)^{-1}$ and can be written in an integral representation in the following way

$$
\Delta=\int_{0}^{\infty} d t e^{-t\left(L_{0}-1\right)}
$$

In this representation it is clear that the divergences in the degenerations (a)-(c) arise from the limit $t \rightarrow \infty$ due to the two lowest lying states namely the $N=0$ and the $N=1$ state, when the boundary values $Y_{1}$ and $Y_{2}$ coincide. For the $N=0$ state this is very similar to the usual tachyon divergence at zero momentum in Neumann theory. For the $N=1$ state the propagator $\Delta$ is infinite if $Y_{1}=Y_{2}$, which is the novel divergence in Dirichlet string theory. Note that while in (a) and (b) the degeneration automatically enforces $Y_{1}=Y_{2}$ on the degenerating strips since there is only one boundary component degenerating, this is not the case for (c) since there are now two boundaries. In Polchinski's scheme these are automatically set equal whereas we will integrate over $Y_{1}$ and $Y_{2}$. The above mentioned position space singularities in $\left(Y_{i}-Y_{j}\right)^{2}$ come from the (c) degeneration as shall be seen later.

To get a more explicit picture the degenerations (a)-(c) can be represented as degenerations on the compact double $\bar{\Sigma}$ (see figure 4) and the well known properties of closed string integrands on the boundary of moduli space [12] can be used. To discuss the case (a) we choose $z_{n} \rightarrow \partial \Sigma_{1}$ where the boundary is at the space time point $Y_{1}$ and a local coordinate system where $I\left(z_{n}\right)=\bar{z}_{n}$ and $\partial \Sigma_{1}$ is represented by the real line. For simplicity consider ground state scattering amplitudes, this will result in an expression which is a special case of $A_{(a)}$ in (26). Writing $z_{n}=x+i \eta$ the divergence in the limit $\eta \rightarrow 0$ comes from the self contraction : $G^{D}\left(z_{n}, z_{n}\right)$ : in $\epsilon_{c l}$. In the path integral normal ordering is equivalent to subtracting out the logarithmic divergence

$$
: G^{D}\left(z_{n}, z_{n}\right):=\lim _{z \rightarrow z_{n}}\left\{G^{D}\left(z, z_{n}\right)-\frac{1}{4 \pi} \ln \left|z-z_{n}\right|^{2}\right\}
$$

If the arguments of the prime form in (17) are close we have

$$
E(z, w)=(z-w)+S(z)(z-w)^{3}+o\left(|z-w|^{4}\right)
$$

Here $S(z)$ is the projective connection on $\bar{\Sigma}$ [26]. The limit $\eta \rightarrow 0$ gives : $G\left(z_{n}, z_{n}\right):=\frac{1}{2 \pi} \ln |\eta|\left[1+o\left(\eta^{2}\right)\right]$ since all other contributions in (17) are of 
order $\eta^{2}$. Setting $k_{n}^{2}=4 / \alpha^{\prime}$ for closed string tachyons the divergence has the form $\exp \left(\epsilon_{c l}\right) \sim \eta^{-2}$. This divergence is due to the level $N=0$ state. To get the contribution from the level $N=1$ state the terms linear in $\eta$ in $\epsilon_{c l}$ have to be extracted. For $i \neq n$ the Green function vanishes when one of the arguments is at the boundary which means that for small $\eta$

$$
G\left(z_{n}, z_{i}\right)=\left.i \eta \partial_{\eta} G^{D}\left(x+i \eta, z_{i}\right)\right|_{\eta=0}+o\left(\eta^{2}\right)
$$

Therefore the term linear in $\eta$ coming from the exponent is

$$
\left.i \sum_{i=1}^{n-1} k_{n} k_{i} \partial_{\eta} G^{D}\left(z_{n}, z_{i}\right)\right|_{z_{i} \epsilon \partial \Sigma_{1}}
$$

Note that the derivative with respect to $\eta$ is the normal derivative with respect to the boundary $\partial \Sigma_{1}$. The Disk amplitude $\left\langle V\left(k_{n}\right) \oint \mathbf{1}\right\rangle_{\text {disk }}$ with one closed string tachyon vertex operator $V\left(k_{n}\right)$ and a $N=0$ boundary vertex operator $\oint \mathbf{1}$ is given by a phase factor $\exp \left(i k_{n} Y_{1}\right)$ which is important for overall momentum conservation. The Disk diagram with one closed string tachyon operator and one insertion of a $N=1$ boundary vertex operator $\oint \partial_{n} X$ is given by $\left\langle V\left(k_{n}\right) \oint \partial_{n} X^{\mu}\right\rangle_{d i s k}$ and is proportional to $k_{n}^{\mu} \exp \left(i k_{n} Y_{1}\right)$. On the other part of the surface $\Sigma$ the insertion of $n-1$ closed string tachyon vertex operators is $\left\langle\prod_{i=1}^{n-1} V\left(k_{i}\right) \oint \partial_{n} X^{\mu}\right\rangle_{\Sigma}=\sum_{i=1}^{n-1} k_{i}^{\mu} \oint d z \partial_{n} G^{D}\left(z, z_{i}\right)$, where \langle\rangle$_{\Sigma}$ denotes the expectation value on the surface $\Sigma$. Putting this all together the divergent part of the amplitude is given by

$$
\begin{gathered}
A_{d i v}^{(a)}=\int d \eta \eta^{-2}\left\langle V_{1} . . V_{n-1} \oint \mathbf{1}\right\rangle_{\Sigma}\left\langle\oint 1 \mathrm{~V}_{\mathrm{n}}\right\rangle_{\mathrm{D}} \\
+\int d \eta \eta^{-1}\left\langle V_{1} . . V_{n-1} \oint \partial_{n} X_{\mu}\right\rangle_{\Sigma}\left\langle\oint \partial_{n} X^{\mu} V_{n}\right\rangle_{D}
\end{gathered}
$$

Note that the level 0 state is just the (integrated) tachyon operator which is inserted in the first term in (35). The $N=0$ divergence is very much like a tachyonic divergence at zero momentum which one encounters in closed string theory. It has been argued [28] that such divergences are due to the failure of the integral representation for the propagator in (30) and that the integrals can be defined by analytic continuation,

$$
\int \frac{d l}{l^{2}}=\lim _{s \rightarrow 0} \int \frac{d l}{l^{2+s}}=-\frac{1}{1+s}=-1
$$


Although subject to ambiguities, this argument supports the conjecture that only really dangerous divergences are logarithmic divergences like $\int d l / l$. In the context of the scattering of massless states the $N=0$ divergence is not there if one considers special helicity amplitudes as shall be seen in the next section.

To discuss the cases (b) and (c) the plumbing fixture parameterization has to be modified in a way which is compatible with the involution $I$. The case (b) corresponds to a pinched zero homology cycle and the case (c) to a pinched b-cycle. The pinching of an a-cycle corresponds to case (d). The construction of the plumbing fixture is as follows [12]. Choose two points $p_{i}$ with $i=1,2$ and coordinate disks $D_{i}=\left\{\left|z_{i}\right|<1\right\}$ on two disconnected surfaces $\Sigma^{(i)}$ for (b) or on one surface $\Sigma$ for (c). For a fixed complex $t$ with $0<|t|<1$ remove smaller disks $D_{i}^{t}=\left\{\left|z_{i}\right|<|t|^{1 / 2}\right\}$ and glue the remaining surfaces and a cylinder $C_{t}=\left\{w:|t|^{1 / 2}<w<|t|^{-1 / 2}\right\}$ together in the following fashion:

$$
w=\left\{\begin{array}{lll}
\frac{t^{\frac{1}{2}}}{z_{1}} & \text { for } & |t|^{\frac{1}{2}}<|w|<1 \\
\frac{z_{2}}{t^{\frac{1}{2}}} & \text { for } & 1<|w|<|t|^{-\frac{1}{2}}
\end{array}\right.
$$

The appropriate coordinates on the moduli space are $d^{2} p_{1}, d^{2} p_{2}, d^{2} t$ and the coordinates of the moduli space of the two separate surfaces. For the open string the moduli space has to be restricted to a real slice. This can be done choosing $t$ to be real and the $p_{i}$ to lie in the appropriate boundary component, i.e. the $p_{i}$ are invariant under $I$. The integration volume element is then given by $d t, d p_{1}, d p_{2}$ where this implies a integration along the boundary components.

To discuss the case (b) behavior of the terms in (10) and (18) under $t \rightarrow 0$ is important, adopting the notation that an index ${ }^{(i)}$ denotes evaluation on the degenerated surface $\Sigma_{i}$. In [12] it is shown that closed string measure (3) factorizes into two parts depending on the modular parameters on $\Sigma_{1}$ and $\Sigma_{2}$ and the divergent part $d^{2} t|t|^{-4}$. The measure on the bordered surface is given by (10) and we see that the divergent part is given by $d t / t^{-2}$. To fix notation we assume that $\Sigma_{1}$ contains the first $k$ homology cycles $\left(a_{i}, b_{i}\right)$ : $i=1, . ., k$ and that $\Sigma_{2}$ contains the rest $\left(a_{1}, b_{i}\right): i=k+1, . ., g$. For a holomorphic differential on the first degenerated surface, i.e. $i \epsilon\{1, . ., k\}$ the 
limiting behavior as $t \rightarrow 0$ is given by

$$
\omega_{i}(z) \rightarrow\left\{\begin{array}{lll}
\omega_{i}^{(1)}(z)+\frac{1}{4} t \omega^{(1)}\left(p_{1}\right) \omega_{p_{1}}^{(1)}(z), & \text { for } & z \epsilon \Sigma^{(1)} \\
\frac{1}{4} t \omega^{(1)}\left(p_{1}\right) \omega_{p_{2}}^{(2)}(z)+O\left(t^{2}\right) & \text { for } \quad z \epsilon \Sigma^{(2)}
\end{array}\right.
$$

and similarly for $i=k+1, . ., g$. Here $\omega_{p_{i}}^{i}$ denotes the abelian differential of the second kind with double poles at $p_{i}$ [25]. It is then clear that the period matrix behaves as

$$
\tau \rightarrow\left\{\begin{array}{cc}
\tau^{(1)} & t \gamma \\
t \gamma & \tau^{(2)}
\end{array}\right\}+O\left(t^{2}\right)
$$

where $\gamma$ is some constant. The behavior of the prime form depends on which part of the surface the arguments lie, namely

$$
\begin{aligned}
& E(z, w) \rightarrow E^{(i)}(z, w)+O(t) \text { if } z, w \epsilon \Sigma^{(i)} \\
& E(z, w) \rightarrow t^{-1 / 2} E^{(1)}\left(z, p_{1}\right) E^{(2)}\left(w, p_{2}\right)+O\left(t^{1 / 2}\right) \text { if } z \epsilon \Sigma_{1}, w \epsilon \Sigma_{2}
\end{aligned}
$$

There is an important difference from the usual open string theory. Substituting (40) into (16) the terms proportional to $\ln |t|$ cancel between the two contributions because of the minus sign in (16). This means that the divergent behavior as $t \rightarrow 0$ is not momentum dependent and there are no poles from intermediate open string states. In this respect the Dirichlet string theory is not a theory of open strings since there are only closed string poles in the scattering amplitudes. The level $N=0$ and $N=1$ contributions arise from the terms in $\epsilon_{c l}$ which are of order $t^{0}$ and $t^{1}$. It is easy to see that for the lowest order $\epsilon_{c l}$ factorizes. If the first $l$ vertex operators lie in $\Sigma_{1}$ and the rest in $\Sigma_{2}$ the exponential energy is given by

$$
\epsilon_{c l}=\sum_{i, j=1}^{l} k_{i} k_{j} G^{(1)}\left(z_{i}, z_{j}\right)+\sum_{i, j=l+1}^{n} k_{i} k_{j} G^{(2)}\left(z_{i}, z_{j}\right)+o(t)
$$

The possible cross terms vanish to this order since $I\left(p_{i}\right)=p_{i}$ and so the contribution of the first terms in (17) cancel. The factorization of the period matrix leads to the cancellation of the second terms in (17) up to this order. The $N=0$ contribution can be written

$$
\int \frac{d t}{t^{2}}\left\langle V\left(z_{1}\right) . . V\left(z_{l}\right) \oint \mathbf{1}\right\rangle_{\Sigma^{(1)}}\left\langle\mathrm{V}\left(\mathrm{z}_{1+1}\right) . . \mathrm{V}\left(\mathrm{z}_{\mathrm{n}}\right) \oint \mathbf{1}\right\rangle_{\Sigma^{(2)}}
$$


The formulas above can be generalized for the $N=1$ contribution and there are also formulas for the degeneration of the differentials and the prime form under the pinching of nonzero homology cycles [26] which are necessary for a similar treatment of case (c). One also has to take the factorization of the measure into account which also gives a nontrivial contribution to the $N=1$ case. In principle one can do this by using the representation of the measure (3) in terms of theta functions and prime forms [12]. Another argument is based on the factorization formulae obtained by Polchinski [34] justifies the formula (26). The behavior of string amplitudes can be investigated by using sewing techniques in CFT [35]. For the correlation function on a closed surface with a fixed conformal structure, a plumbing fixture construction gives the factorization

$$
\left\langle V_{1} . . V_{n}\right\rangle_{\Sigma}=\sum_{i} t^{h_{i}} \overline{\bar{t}_{i}}\left\langle V_{1} . . V_{l} A_{i}\right\rangle_{\Sigma_{1}}\left\langle V_{l+1} . . V_{n} A^{i}\right\rangle_{\Sigma_{2}}
$$

This amounts to an insertion of a complete set of states at the punctured surfaces $\Sigma_{1}$ and $\Sigma_{2}$ where $h_{i}$ and $\bar{h}_{i}$ are the left and right conformal dimensions of the state $A_{i}$. In string theory one has to integrate over all possible comformal structures, i.e. over the moduli space. In the formalism with $b c$ ghosts and ghost insertions of (파) the appropriate thing to do is to find the Beltrami differential corresponding to the plumbing fixture coordinates $t, \bar{t}$, pair them with the ghost zero modes and factorize over the complete CFT including the ghost states. In the case of a zero (dividing) cycle the other coordinates on the moduli space are the modular parameters $m^{(i)}$ and the puncture $p^{(i)}$ on the surface $\Sigma_{i}$,with $i=1,2$. In the case of a nonzero (nondividing) cycle the moduli parameters are conveniently chosen to be $m$ of $\Sigma^{\prime}$ and the two punctures $p^{(i)}$ on $\Sigma^{\prime}$. In order to factorize on a complete set of states choose a coordinate system $z_{i}$ at $p^{(i)}$ to get a Hilbert space description of the CFT. The insertion of a complete set of states in the path integral is given by [34.

$$
\sum_{i} t^{h_{i}-1} \bar{t}^{\overline{h_{i}}-1} b_{0}^{(1)} \bar{b}_{0}^{(1)}\left|\Phi_{i}\right\rangle^{(1)}\left|\Phi^{i}\right\rangle^{(2)}
$$

The ghost insertion is $(t \bar{t})^{-1} b_{0} \bar{b}_{0}$ corresponding to the $t, \bar{t}$ coordinates on moduli space. $|\Phi\rangle^{(i)}$ denotes the insertion of a state $|\Phi\rangle$ in the Hilbert space of states at the puncture $p_{i}$. The sum is taken over all states of the combined $X, b, c$ system. There are some subtleties due to the fact that to define 
this factorization a choice of a coordinate system at the punctures has to be made, see [34] for details. This can be applied to the degeneration of bordered surfaces discussed in section 2.3, by noting that closed string factorization implies the open string result via the doubling procedure. For the generic example of the half plane geometry the open string conformal field theory can be expressed by the closed string (bulk) CFT by identifying $X(\bar{z})= \pm \bar{X}(z)$ and just keeping the chiral half of the theory defined now in the plane. The different signs correspond to Neumann and Dirichlet boundary conditions. This local description works for more complicated topologies since the sewing is locally described by coordinate systems at the two punctures $p_{i}$. For the Dirichlet boundary conditions one has to be careful about the zero modes, the states on which one factorizes are automatically at zero momentum, as is clear from the discussion at the end of section 2.2. The factorization on the level one state $N=1$ is then given by (see appendix B for some conventions for the ghosts)

$$
t^{\left(Y_{2}-Y_{1}\right)^{2}-1}\left\{-\eta_{\mu \nu} a_{-1}^{(1) \mu} a_{-1}^{(2) \nu}+c_{-1}^{(1)} b_{-1}^{(2)}-b_{-1}^{(1)} c_{-1}^{(2)}\right\} c_{1}^{(1)}|\downarrow\rangle^{(1)} c_{1}^{(2)}|\downarrow\rangle^{(2)}
$$

The factor of $\left(Y_{1}-Y_{2}\right)^{2}$ comes from $L_{0}$ and reflects the fact that the conformal weight of the open string states do not depend on the momentum but on the boundary positions. Due to the fact that the expression is at zero momentum the $a_{-1}$ mode can be separated from the rest which can be written as a BRST exact state, hence (45) is given by ( setting $Y_{1}=Y_{2}$ )

$$
\begin{gathered}
t^{-1}\left\{-\eta_{\mu \nu} a_{-1}^{(1) \mu} a_{-1}^{(2) \nu}|\downarrow\rangle^{(1)} c_{1}^{(2)}|\downarrow\rangle^{(2)}\right. \\
\left.+\left(Q_{(1)}+Q_{(2)}\right)\left[b_{-1}^{(2)}|\chi\rangle^{(1)}|\downarrow\rangle^{(2)}-b_{-1}^{(1)}|\chi\rangle^{(2)}|\downarrow\rangle^{(1)}\right]\right\}
\end{gathered}
$$

Here the BRST operator on the surface $\Sigma_{i}$ is denoted $Q^{(i)}$ and $c_{-1}^{(i)}|\downarrow\rangle^{(i)}=$ $Q^{(i)}|\chi\rangle^{(i)}$, this is possible because $k^{\mu}=0$ and terms $a_{-1}^{(i) \mu} k_{\mu}|\chi\rangle^{(i)}$ vanish. This is remarkable since in the usual case the ghosts are needed to cancel unphysical polarizations, here the Dirichlet boundary condition automatically enforces $k=0$ and the BRST cohomolgy decouples the ghost states and leaves the level one state. The appropriate vertex operator insertion corresponding to $a_{-1}^{\mu}$ is given by $\oint \partial_{n} X^{\mu}$ in the case of Dirichlet boundary conditions.

The properties of the period matrix under degeneration of a nonzero cycle can be used to show that this boundary of moduli space is responsible for the 
divergences in position space for the Dirichlet partition function mentioned earlier. If for example $b_{g}$-cycle is pinched the period matrix behaves as

$$
\tau \rightarrow\left\{\begin{array}{cc}
\tau^{\prime} & \oint_{p_{1}}^{p_{2}} \omega \\
\left(\oint_{p_{1}}^{p_{2}} \omega\right)^{t} & \ln t
\end{array}\right\}+O(t)
$$

Here $\tau^{\prime}$ is the period matrix on the genus $g-1$ surface with the punctures $p_{1}$ and $p_{2}$ where the cycle was pinched, the $\omega_{i}$ are the remaining $g-1$ holomorphic differentials. Putting this into (20) the $\ln t$ part of the period matrix gives a term in $\epsilon_{c l}$ which behaves as $\exp \left(\ln t\left(Y_{g-1}-Y_{g}\right)^{2}\right)$. This term is responsible for the singularities in $\left(Y_{g-1}-Y_{g}\right)^{2}$ in the limit $t \rightarrow 0$. The above mentioned singularities outside, on and inside the lightcone are obtained when the singular behavior of the measure on moduli space is also taken into account 33] which contributes $\exp (\ln t(-1+n))$, where $n$ is a positive integer. The presence of singularities away from the lightcone is unlike normal field theoretic amplitudes and this is a decidedly stringy effect [6]. Other pinches of nonzero cycles give the singularities in different combinations of the $Y_{i}$. In section 4 this is discussed in detail for the case of the annulus. Multiple pinches should lead to cuts in position space, and this gives a similar picture to the analyticity of scattering amplitudes in momentum space for the Neumann theory, but now in position space.

\section{One Dirichlet boundary insertion}

The inclusion of one Dirichlet boundary in the world sheet yields the topology of the half plane. The general method of the last section is now just the method of images on the half plane, where the Green function for the complex plane $-1 / 4 \ln |z-w|$ gives for the Dirichlet Green function

$$
G^{D}(z, w)=-\frac{1}{4 \pi}\left\{\ln |z-w|^{2}-\ln |z-\bar{w}|^{2}\right\}
$$

The classical solution is a constant $X_{c l}^{\mu}=Y^{\mu}$, which upon integration in (18) leads to momentum conservation in scattering amplitudes. The scattering amplitude for arbitrary closed string vertex operators is then given by

$$
\Gamma\left(k_{1}, \cdots, k_{n}\right)=\int \frac{\prod_{i=k}^{n} d^{2} z_{k}}{\operatorname{Vol}(S L(2, R))}\langle\text { contractions }\rangle \exp \left\{\epsilon_{c l}\right\}
$$


Where the exponential energy is given by

$$
\epsilon_{c l}=\sum_{i>j}^{n} k_{i} k_{j}\left\{\ln \left|z_{i}-z_{j}\right|^{2}-\ln \left|z_{i}-\bar{z}_{j}\right|^{2}\right\}+\sum_{i=1}^{n} k_{i}^{2} \ln \left|z_{i}-\bar{z}_{i}\right|^{2}
$$

the <contractions > denote contractions coming from the tensor vertex operators. $\operatorname{Vol}(\mathrm{Sl}(2, \mathrm{R}))$ denotes the volume of the group generated by the conformal Killing vectors, which can be used to fix $z_{1}=i$ and to set $\operatorname{Re}\left(z_{2}\right)=0$. The second sum in (50) comes from the regularization of the Green function at coincident points. The form of the contractions depends on the vertex operators considered. The half plane has no modular parameter so the only divergent contribution can come from case (a) in the general analysis, namely, when some of the $z_{i}$ approach the boundary, i.e. $\operatorname{Im}\left(z_{i}\right) \rightarrow 0$. The analysis of the divergences in this limit is only a special case of the general analysis of case (a).

As discussed earlier the high energy fixed angle scattering amplitude is dominated by the region where $\epsilon_{c l}$ in (50) vanishes. However this coincides with the region where the integral diverges due to the level zero and and level one open string states. The naive result is that after fixing $z_{1}$ with the Moebius group invariance the integration over deviations $z_{2}=i \delta y_{2}, z_{3}=$ $x_{3}+i \delta y_{3}, z_{4}=x_{4}+i \delta y_{4}$ gives a power behavior of $s^{-3}$ since the terms in $\epsilon_{c l}$ are linear in the deviations. The presence of divergences for external tachyons invalidates the analysis.

In order to avoid the problematic divergences we turn to consider amplitudes with external massless tensor states. Contractions of the standard massless tensor vertex operators

$$
V(k, \xi ; z)=: \xi_{\mu \nu} \partial_{z} X^{\mu}(z) \partial_{\bar{z}} \overline{X^{\nu}(z)} e^{i k_{\rho} X(z, \bar{z})^{\rho}}:
$$

give rise to the following terms

$$
\begin{aligned}
\left\langle\partial_{z} X^{\mu}(z) \partial_{w} X^{\nu}(w)\right\rangle & =\delta^{\mu \nu} \frac{1}{(z-w)^{2}} \\
\left\langle\partial_{z} X^{\mu}(z) \partial_{\bar{w}} \overline{X^{\nu}(w)}\right\rangle & =\delta^{\mu \nu} \frac{1}{(z-\bar{w})^{2}}
\end{aligned}
$$

We can check whether there are any special contractions i.e. special helicity amplitudes of the tensors where the infinity above disappears. This is indeed 
the case for the following 4-particle scattering amplitude as noted in [6]

$$
\xi_{\mu_{1} \nu_{1}}^{1} \xi_{\mu_{2} \nu_{2}}^{2} \xi_{\mu_{3} \nu_{3}}^{3} \xi_{\mu_{4} \nu_{4}}^{4}\left\langle\partial X^{\mu_{2}} \overline{\partial X^{\nu_{3}}}\right\rangle\left\langle\partial X^{\mu_{3}} \overline{\partial X^{\nu 4}}\right\rangle\left\langle\partial X^{\mu_{4}} \overline{\partial X^{\nu_{1}}}\right\rangle\left\langle\partial X^{\mu_{1}} \overline{\partial X^{\nu_{2}}}\right\rangle
$$

To see that such amplitudes are the only ones which are finite define the following scaling variable $\eta$, where either one, two or three of $y_{2}, z_{3}, z_{4}$ are scaled with $\eta$, defining $y_{2}=i \eta, z_{3}=\eta \xi_{3}$ and/or $z_{4}=\eta \xi_{4}$. If two of the variables are scaled there is a factor of $\eta^{2}$ from the measure, if three are scaled the contribution is $\eta^{4}$. It is now easy to see that there is a total of four contractions for two particle scattering and that only the 'cyclic' type (52) is finite under these scalings.

There is a general reason that both the $N=0$ scalar and $N=1$ vector open string state decouple in an arbitrary diagram contributing to this particular helicity amplitude. This can be seen by cutting a diagram where one, two or three vertex operators approach the boundary. In the case of cyclic contractions there are at least two Lorentz indices contracted between the two halves of the diagram i.e. the intermediate propagating state has to have a tensorial structure. However the open string $N=0$ and $N=1$ states couple to scalars and derivatives of scalars respectively, and therefore decouple from the special helicity amplitudes (52). Hence there can not be a divergence when the vertex operators approach the boundary.

It is easy to see that the considerations are not limited to two particle scattering and that in general for $n$ vertex operator insertions the 'cyclic' amplitudes yield no divergence from the asymptotic region. The high energy fixed angle scattering behavior for one Dirichlet boundary insertion is governed by the integration over the deviations of $y_{2}, z_{3}, . ., z_{n}$ from the real line which gives $s^{-(n-1)}$ for $n$ vertex operator insertions.

\section{Two Dirichlet boundary insertions}

A world sheet with two Dirichlet boundaries and no handles is the annulus. The annulus has one real modular parameter (the ratio between the radii). The torus with purely imaginary modular parameter $\tau=i l$ is represented by a fundamental cell in the z-plane

$$
0<\operatorname{Im}(z)<l: 0<\operatorname{Re}(z)<1
$$


The holomorphic differential is $\omega=d z$ and the period matrix is given by the modular parameter $\tau$. The annulus can be represented as the quotient under the Involution $I(z)=\bar{z}$, and the boundaries are given by $\partial \Sigma_{1}=\{\operatorname{Im}(z)=0\}$ and $\partial \Sigma_{2}=\{\operatorname{Im}(z)=1 / 2\}$. (See Figure 5) The measure on the moduli space of the torus is [17

$$
\int \frac{d^{2} \tau}{|\operatorname{Im}(\tau)|^{14}}|\eta(\tau)|^{-48}
$$

The presence of conformal killing vectors on the torus and annulus has to be included in the calculation of the measure and the measure on the annulus is not given by (10), but 14

$$
\int \frac{d l}{l^{13}} n(i l)^{-24}
$$

Where the $\eta$-function is given by $\eta(i l)=e^{-\pi l / 12} \prod\left(1-e^{-\pi n l}\right)$. The Green function on the torus is well known to be

$$
G_{\bar{\Sigma}}=-\frac{1}{4 \pi} \ln \left|\frac{\theta_{1}(z-w \mid \tau)}{\theta_{1} \prime(0 \mid \tau)}\right|^{2}+\frac{1}{2} \frac{\{\operatorname{Im}(z-w)\}^{2}}{\operatorname{Im} \tau}
$$

the second term comes from $\frac{1}{2} \operatorname{Im} \int_{z}^{w} d z \operatorname{Im} \tau^{-1} \int_{z}^{w} d z=\{\operatorname{Im}(z-w)\}^{2} / \operatorname{Im} \tau$. the Dirichlet Green function on $\Sigma$ can then be expressed using (24) as

$$
G^{D}(z, w)=\frac{1}{4 \pi} \ln \left|\frac{\theta_{1}(z-w \mid \tau)}{\theta_{1}(z-\bar{w} \mid \tau)}\right|^{2}-2 \frac{(z-\bar{z})(w-\bar{w})}{\operatorname{Im} \tau}
$$

In this case $X_{c l}(z)=Y_{1}-\left(Y_{2}-Y_{1}\right) / \operatorname{Im}(\tau)$ is the classical part in (12). The integral over $Y_{1}$ and $Y_{2}$ is done as in (23) using the relations

$$
\begin{aligned}
b & =\sum_{i} k_{i} \int_{0}^{1} \partial_{w} G^{D}\left(z_{i}, w\right) \\
& =\sum_{i} k_{i} \frac{\left(z_{i}-\bar{z}_{i}\right)}{\operatorname{Im} \tau}
\end{aligned}
$$

The second term in (57) is cancelled by the contribution coming from (58) inserted in (24) and the modified Green function is given by

$$
\hat{G}^{D}(z, w)=\frac{1}{4 \pi} \ln \left|\frac{\theta_{1}(z-w \mid \tau)}{\theta_{1}(z-\bar{w} \mid \tau)}\right|^{2}
$$


which coincides with the Green function found in [8] obtained with different methods. The integration produces powers of $\operatorname{Im}(\tau)$ so that the measure (55) is given by

$$
\int d \ln (i l)^{-24}
$$

\subsection{High-energy fixed angle scattering of massless ten- sor states}

High energy fixed angle scattering is dominated by the contributions of the endpoints at which the vertices are close to the boundary. The behavior of the Green function $\hat{G}^{D}(z, w)$ is given by (we denote the modified Green function of section 4 by $\mathrm{G}$ )

$$
\begin{array}{ll}
z \epsilon \partial \Sigma_{1}, w \epsilon \partial \Sigma_{1}: & G^{D}=0, \partial_{z} G^{D}=0, \partial_{z} \partial_{w} G^{D} \neq 0 \\
z \epsilon \partial \Sigma_{1}, w \epsilon \partial \Sigma_{2} & : \quad G^{D}=0, \partial_{z} G^{D}=\frac{i \tau}{2}, \partial_{z} \partial_{w} G^{D} \neq 0 \\
z \epsilon \partial \Sigma_{2}, w \epsilon \partial \Sigma_{2} & : \quad G^{D}=\frac{1}{2}, \partial_{z} G^{D}=\frac{i \tau}{2}, \partial_{z} \partial_{w} G^{D} \neq 0
\end{array}
$$

Note that there seems to be an asymmetry between the two boundaries in the formula above, this is not so in the scattering amplitudes because of momentum conservation. As in the case of the one boundary insertion the scattering amplitudes are constructed from the Green function the 'exponential energy' is given by

$$
\epsilon_{c l}=\pi \alpha^{\prime} \sum_{i \neq j} G^{D}\left(z_{i}, z_{j}\right) k_{i} k_{j}
$$

In order to analyze the behavior of $\epsilon$ when all the $z_{i}$ are near the boundary $\partial \Sigma_{1}$. write $z_{i}=x_{i}+i \delta y_{i}$ and make a Taylor expansion at $\delta y_{i}=0$. Using the fact that on the boundary $z_{i}=\bar{z}_{i}$ it is easy to see that the only term up to order $o\left(\delta y^{2}\right)$ is

$$
G^{D}\left(z_{i}, z_{j}\right)=\frac{1}{2} \partial_{y_{i}} \partial_{y_{j}} G^{D}\left(x_{i}, x_{j}\right) \delta y_{i} \delta y_{j}+o\left(\delta y^{2}\right)
$$

Using complex coordinates $\frac{\partial}{\partial y}=i \frac{\partial}{\partial z}-i \frac{\partial}{\partial \bar{z}}$ and defining

$$
h(z, w)=-\frac{1}{4 \pi} \frac{\theta_{1}^{\prime \prime}(z-w \mid \tau) \theta_{1}(z-w \mid \tau)-\theta_{1}^{\prime}(z-w \mid \tau)^{2}}{\theta_{1}(z-w \mid \tau)^{2}}
$$


Defining $\partial_{y_{i}} \partial_{y_{j}} G\left(x_{i}, x_{j}\right)=H_{i j}$ we see that

$$
\begin{aligned}
H_{i j} & =-4 h\left(x_{i}, x_{j}\right) \\
& =-\pi\left\{\frac{1}{\sin ^{2} \pi\left(x_{i}-x_{j}\right)}+8 \sum_{n} n \frac{q^{2 n}}{1-q^{2 n}} \cos 2 n \pi\left(x_{i}-x_{j}\right)\right\}
\end{aligned}
$$

where the following identity [27] for theta functions was used

$$
\frac{\theta_{1}^{\prime}(z \mid \tau)}{\theta_{1}(z \mid \tau)}=\pi \cot \pi z+4 \pi \sum_{n} \frac{q^{2 n}}{1-q^{2 n}} \sin 2 m \pi z
$$

It is now simple to write $\epsilon_{c l}$ as a power series in $\delta y_{i}$ for $i=1, \cdots, n$. In the fixed angle high energy limit which we want to consider, all factors $k_{i} k_{j}$ become large. A end point integration of the deviations $\delta y_{i}$ gives the asymptotic behavior of the scattering amplitude. For the two particle scattering with the Mandelstam variables $s=-\left(k_{1}+k_{2}\right)^{2}, t=-\left(k_{1}+k_{3}\right)^{2}, u=-\left(k_{1}+k_{4}\right)^{2}$

$$
\begin{aligned}
\epsilon_{c l}= & s\left\{H_{12} \delta y_{1} \delta y_{2}+H_{34} \delta y_{3} \delta y_{4}\right\}+t\left\{H_{13} \delta y_{1} \delta y_{3}+H_{24} \delta y_{2} \delta y_{4}\right\} \\
& +u\left\{H_{14} \delta y_{1} \delta y_{4}+H_{23} \delta y_{2} \delta y_{3}\right\} \\
= & \sum_{i j} M_{i j} \delta y_{i} \delta y_{j}
\end{aligned}
$$

The Gaussian integration gives a factor $(\operatorname{det} M)^{-1 / 2}$. Evaluating the determinant and noting that for $s \rightarrow \infty$ in the fixed angle limit $-s / t=\sin ^{2} \Phi / 2$ and $-u / s=\cos ^{2} \Phi / 2$ where $\Phi$ is the centre of mass frame scattering angle, the determinant is given by

$$
\begin{aligned}
\operatorname{det}(M)= & s^{4}\left\{\left(H_{12} H_{34}\right)^{2}+\left(H_{13} H_{24}\right)^{2} \sin ^{8} \frac{\Phi}{2}+\left(H_{14} H_{23}\right)^{2} \cos ^{8} \frac{\Phi}{2}\right. \\
& +2 H_{12} H_{34} H_{23} H_{14}+2 H_{12} H_{34} H_{13} H_{24} \cos ^{4} \frac{\Phi}{2} \\
& \left.-2 H_{23} H_{13} H_{14} H_{24} \cos ^{4} \frac{\Phi}{2} \sin ^{4} \frac{\Phi}{2}\right\}
\end{aligned}
$$

The term in the brackets depends only on the scattering angle. Therefore we expect that the leading asymptotic behavior of the scattering amplitude is $s^{-2}$. The explanation for the smaller power in comparison to the disk topology lies in the fact that for the disk there is the $S L(2, R)$ conformal Killing group, which allowed to fix one of the vertices at the origin of the 
disk, so there could only be three vertices touching the boundary. If one vertex touches one boundary and three are close to the other $\epsilon_{c l}$ is linear in the deviations and the asymptotic behavior is $s^{-3}$ which is subleading. If two vertices are on one boundary and two are on the other $\epsilon_{c l}$ does not vanish and the amplitude is exponentially suppresed.

\subsection{Boundary of moduli space}

This analysis is naively correct but one has to consider the effect of possible divergences coming from the boundary of moduli space. The cases to consider are (a): vertices going to $\partial \Sigma_{1}$ or $\partial \Sigma_{2}$, case(c): the limit $l \rightarrow 0$ and case (d): the limit $l \rightarrow \infty$.

(a) As for the disk the tachyon amplitudes are meaningless due to a boundary divergence since the self-contraction : $G^{D}(z, z)$ : is defined by

$$
\lim _{z \rightarrow w}\left\{G^{D}(z, w)-\frac{1}{4 \pi} \ln |z-w|^{2}\right\}=\frac{1}{4 \pi} \ln \left|\frac{\theta_{1}^{\prime}(0 \mid \tau)}{\theta_{1}(z-\bar{z} \mid \tau)}\right|^{2}
$$

and this behaves like $|z-\bar{z}|^{2}$ if $\mathrm{z}$ is near the boundary. As we shall see the 'cyclic' tensor-amplitudes like (52) circumvent these divergences.

The contractions for the tensor-amplitudes are given by (64)

$$
\begin{aligned}
\left\langle\partial_{z} X \partial_{w} X\right\rangle & =h(z, w) \\
\left\langle\partial_{z} X \overline{\partial_{w} X}\right\rangle & =h(z, \bar{w})
\end{aligned}
$$

To consider the finiteness of this amplitude we can repeat the analysis of section 3 for case (a) which applies also for the annulus (since near a particular boundary the other boundaries can be ignored). But now all four vertices can come close to one boundary. This region of moduli space can be investigated defining new variables

$$
z_{1}=x_{1}+i \eta, z_{2}=x_{1}+\eta \xi_{2}, z_{3}=x_{1}+\eta \xi_{3}, z_{4}=x_{1}+\eta \xi_{4}
$$

The measure behaves as $\prod_{i=1}^{3} d^{2} z_{i}=\eta^{6} d \eta d x_{1} \prod_{i=2}^{4} d^{2} \xi_{i}$ in terms of the new integration variables. The Green function behaves as

$$
G^{D}\left(z_{i}, z_{j}\right)=-1 / 4 \pi \ln \left|\frac{\xi_{i}-\xi_{j}}{\xi_{i}-\bar{\xi}_{i}}\right|^{2}+o\left(\eta^{2}\right)
$$


(note that $\xi_{1}=i$ and that there is no linear term in $\eta$ ). In contrast to the analysis of the half plane the four vertices give a contribution which is singular, since there is a $\eta^{-8}$ coming from the contractions of (52) $\int d \eta \eta^{-2} A_{1}$. The divergent part $A_{1}$ is given by

$$
\begin{aligned}
A_{1}= & \int d l \mu(l) \int \prod_{i=1}^{4} d^{2} \xi_{i} \delta^{2}\left(\xi_{1}-i\right) \frac{1}{\left(\xi_{1}-\bar{\xi}_{2}\right)^{2}} \frac{1}{\left(\xi_{2}-\bar{\xi}_{3}\right)^{2}} \\
& \frac{1}{\left(\xi_{3}-\bar{\xi}_{4}\right)^{2}} \frac{1}{\left(\xi_{4}-\bar{\xi}_{1}\right)^{2}} \exp \left\{\sum_{i \neq j} k_{i} k_{j} \ln \left|\frac{\xi_{i}-\xi_{j}}{\xi_{i}-\bar{\xi}_{j}}\right|^{2}\right\}
\end{aligned}
$$

$\mu(l)$ is given by (60). Note that $A_{1}$ is very similar to the amplitude for one boundary insertion. This means that the high energy fixed angle behavior in the limit in which all four vertices come together is divergent as $\int d \eta \eta^{-2}$. The coefficient multiplying this divergence has a $s^{-3}$ dependence, hence it is not a leading contribution. A sensible regularization should not spoil this feature. This divergence comes from the presence of the open string $N=0$ state as explained above. There is no divergence coming from the $N=1$ state since the next order term in expanding the Green function is quadratic in $\eta$. This is easy to explain since the $N=1$ state couples to the momenta flowing through the boundary, when four vertices come together the degeneration in case (a) leads to a disk with four vertices coupling to the rest of the diagram with no vertices at all, so that zero momentum flows through the boundary connecting the two parts of the diagram because of momentum conservation.

(c) The limit $l \rightarrow 0$ corresponds to the limit in which the radii of the annulus coincide. To investigate this boundary of moduli space it is useful to make a modular transformation with $i l^{\prime}=\tau^{\prime}$

$$
\tau^{\prime}=-\frac{1}{\tau} ; \zeta_{z}=-\frac{z}{\tau}
$$

The fundamental cell for the annulus is mapped into $0<\operatorname{Re}(\zeta)<1 / 2$, $0<\operatorname{Im}(\zeta)<l^{\prime}$ (see Figure 5). The transformed terms can be expressed using the well known properties of the $\eta$-function and $\theta$-function under modular transformations. The resulting measure is

$$
\int d l \eta(i l)^{-24}=\int \frac{d l^{\prime}}{l^{\prime 14}} \eta\left(i l^{\prime}\right)^{-24}
$$


It can be seen that this limit is also responsible for the singularities in the Dirichlet partition function $A\left(Y_{1}-Y_{2}\right)$ for two boundaries. In this case the general formula (21) reads in the new variables

$$
A\left(Y_{1}-Y_{2}\right)=\int_{0}^{\infty} \frac{d l^{\prime}}{l^{\prime}} \eta\left(i l^{\prime}\right)^{-24} \exp \left\{-\frac{1}{2 \pi \alpha^{\prime}} l^{\prime}\left(Y_{1}-Y_{2}\right)^{2}\right\}
$$

In the limit $l^{\prime} \rightarrow \infty$ expanding the $\eta$-function in powers of $\exp \left(2 \pi l^{\prime}\right)$ and get $\eta\left(i l^{\prime}\right)^{-24} \rightarrow \exp \left(2 \pi l^{\prime}\right)\left(1+o\left[\exp \left(-2 \pi l^{\prime}\right)\right]\right.$. There are logarithmic divergences for certain values of $\left(Y_{2}-Y_{1}\right)^{2}$ namely for $\left(Y_{2}-Y_{1}\right)^{2}=4 \pi^{2} \alpha^{\prime}(1-N)$ where $N$ is an non negative integer. The $N=0$ term is a position space singularity outside the lightcone and the $N=1$ is on the lightcone. The terms with $N>1$ give an infinite tower of singularities inside the light cone. The Green function is written in terms of the new variables using the well known imaginary transformation of Jacobi [27]

$$
\theta_{1}\left(-\frac{z}{\tau} \mid-\frac{1}{\tau}\right)=-i(-i \tau)^{\frac{1}{2}} \exp \left(\frac{i \pi z^{2}}{\tau}\right) \theta_{1}(z \mid \tau)
$$

The Green function (59) written in the new arguments is then easily calculated,

$$
G^{D}\left(\zeta_{z}, \zeta_{w}\right)=-\frac{2 i}{\tau^{\prime}} \operatorname{Re}\left(\zeta_{z}\right) \operatorname{Re}\left(\zeta_{w}\right)-\frac{1}{4 \pi} \ln \left|\frac{\theta_{1}\left(\zeta_{z}-\zeta_{w} \mid \tau^{\prime}\right)}{\theta_{1}\left(\zeta_{z}+\bar{\zeta}_{w} \mid \tau^{\prime}\right)}\right|^{2}
$$

Note the change of sign in the theta function in the denominator which is due to the fact that $\bar{\tau}=-\tau$. To discuss the high energy fixed angle scattering it is convenient to make yet another change of variables. First map the fundamental cell onto the seminannular region with an exponential map $\sigma_{i}=\exp \left(2 \pi \zeta_{i}\right)$ and then introduce new variables $x_{i}$ instead of the $\sigma_{i}, i=$ $1, . ., n$ (see figure 5 )

$$
\begin{aligned}
\sigma_{1} & =x_{1} \\
\sigma_{2} & =x_{1} x_{2} \\
& : \\
\sigma_{n} & =x_{1} . . x_{n}
\end{aligned}
$$

The rotational conformal Killing symmetry of the integrand ( in the $\zeta$ plane this amounts to the invariance of the integrand under imaginary translations) 
can be used to fix $\sigma_{n}=|w|$. With the exponentiated modular parameter $|w|=\exp \left(-2 \pi l^{\prime}\right)$. The region $\left\{x_{i}:\left|x_{i}\right|<1, \operatorname{Im}\left(x_{i}\right)>0, i=1, . . n\right\}$ covers the moduli space of the semiannular parameterization where the $\sigma_{i}$ are ordered, i.e. $\left|\sigma_{1}\right|>\left|\sigma_{2}\right|>\ldots>\left|\sigma_{n}\right|$. To cover the whole moduli space all orderings of the $\sigma_{i}$ in (81) have to be considered. The Jacobian for this change of variables gives

$$
\int \frac{d l}{l^{14}} \eta(i l)^{-24} \prod_{i=1}^{n} d^{2} \zeta_{i}=\prod_{i=1}^{n} d^{2} x_{i}|w|^{-3} \prod_{k=1}^{\infty}\left(1-|w|^{k}\right)^{-24} \ln |w|^{-14}
$$

The case (c) corresponds to $|w| \rightarrow 0$. The new variables are useful since one can distinguish between different limits depending on which and how many of the $x_{i}$ go to zero. The $x_{i}$ are the string equivalent of Schwinger parameters in ordinary field theory. The limit in which one of them vanishes corresponds to a pinching of an internal propagator (30). This becomes clear if the annulus amplitude is constructed in an operator approach as a trace of vertex operators and propagators $\operatorname{Tr}(V \Delta . . V \Delta)$ and the parameterization (81) is used 31.

The arguments which appear in the Green function can be written in terms of the $\xi_{i}$ for $i>j$.

$$
\begin{aligned}
\xi_{i} & =\frac{1}{2 \pi i} \ln \left(x_{1} . . x_{i}\right) \\
\xi_{i}-\xi_{j} & =\frac{1}{2 \pi i} \ln \left(\frac{x_{1} . . x_{i}}{x_{1} . x_{j}}\right) \\
\xi_{i}+\bar{\xi}_{j} & =\frac{1}{2 \pi i} \ln \left(\frac{x_{1} . x_{i}}{\bar{x}_{1} . . \bar{x}_{j}}\right)
\end{aligned}
$$

In the limit $w \rightarrow 0$ some of the $\left|x_{i}\right| \rightarrow 0$. The first term of the Green function (80) can be dominated by a finite, $w$ independent contribution from the second term. To see this consider the expansion of $\theta_{1}$ [27]

$$
\theta_{1}(\xi \mid \tau)=C \sin \pi \xi \prod_{n=1}^{\infty}\left(1-2 w^{n} \cos 2 \pi \xi+w^{2 n}\right)
$$

Here $C$ is a $w$ dependent term which cancels between the two $\theta$-functions in the Green function. In the limit under consideration only $\sin \xi$ and the $n=1$ factor in (84) contribute. They appear in the expression for the Green 
function in the following ratios

$$
\begin{aligned}
\left|\frac{\sin \pi\left(\xi_{i}-\xi_{j}\right)}{\sin \pi\left(\xi_{i}+\bar{\xi}_{j}\right)}\right|^{2} & =\left|\frac{1-\frac{x_{1} . x_{i}}{x_{1} \ldots x_{j}}}{1-\frac{x_{1} . x_{i}}{\overline{x_{1} . . . x_{j}}}}\right|^{2} \text { if } \frac{x_{1} . . x_{i}}{x_{1} . . x_{j}} \neq 0 \\
\left|\frac{1-w \cos 2 \pi\left(\xi_{i}-\xi_{j}\right)}{1-w \cos 2 \pi\left(\xi_{i}+\bar{\xi}_{j}\right)}\right|^{2} & =\left|\frac{1-w \frac{x_{1} . x_{j}}{x_{1} . . x_{i}}}{1-w \frac{x_{1} . x_{j}}{\overline{x_{1} . . . x_{i}}}}\right|^{2} \text { if } w \frac{x_{1} . . x_{i}}{x_{1} . . x_{j}} \neq 0
\end{aligned}
$$

In all other cases the ration of $\theta$ functions is one in this limit and the second term in (80) vanishes. Writing $\epsilon_{c l}=\epsilon_{-1}+\epsilon_{0}$ up to terms vanishing in linear order of $\left|x_{i}\right|$. Firstly $\epsilon_{-1}$ is coming from the first term of (80).

$$
\epsilon_{-1}=\sum_{i j} k_{i} k_{j} \frac{1}{2 \pi^{2} \ln w} \ln \left(\frac{x_{1} . . x_{i}}{\bar{x}_{1} . . \bar{x}_{i}}\right) \ln \left(\frac{x_{1} . . x_{j}}{\bar{x}_{1} . . \bar{x}_{j}}\right)
$$

Secondly $\epsilon_{0}$ is coming from the nonvanishing terms in (86). Consider the example in which $x_{1} \rightarrow 0$ and all other $x_{i}$ finite. Writing $x_{1}=\eta e^{i \phi_{1}}$ with $\eta \rightarrow 0$

$$
\begin{aligned}
\epsilon_{0} & =k_{1} k_{2} \ln \left|\frac{1-x_{2}}{1-x_{1} x_{2} / \bar{x}_{1}} \frac{1-x_{4}}{1-\left(x_{1} x_{2} x_{3} x_{4}\right) / \overline{\left(x_{1} x_{2} x_{3}\right)}}\right|^{2} \\
& +k_{1} k_{3} \ln \left|\frac{1-x_{2} x_{3}}{1-x_{1} x_{2} x_{3} / \bar{x}_{1}} \frac{1-x_{3} x_{4}}{1-\left(x_{1} x_{2} x_{3} x_{4}\right) / \overline{\left(x_{1} x_{2}\right)}}\right|^{2} \\
& +k_{1} k_{4} \ln \left|\frac{1-x_{2} x_{3} x_{4}}{1-x_{1} x_{2} x_{3} x_{4} / \bar{x}_{1}} \frac{1-x_{3}}{1-\left(x_{1} x_{2} x_{3}\right) / \overline{\left(x_{1} x_{2}\right)}}\right|^{2} \\
& +o(\eta)
\end{aligned}
$$

The first three terms in (88) depend only on $x_{1} / \overline{x_{1}}$ and are independent of $\eta$. Denoting $x_{i}=r_{i} e^{i \phi_{1}}$ for $i=2,3,4$ it can be easily seen that the $\eta$ independent part of $\epsilon_{0}$ has still the property we met in the general analysis of section 4.1 namely that it vanishes when the vertex operator positions approach the boundaries. The boundary $\partial \Sigma_{1}$ corresponds to $\phi_{i}=0$ and $\partial \Sigma_{2}$ corresponds to $\phi_{i}=\pi$ for all $i$ (see figure 5 ). It can also be shown that the $\eta$-independent part of $\epsilon_{0}$ is quadratic in the deviations $\delta \phi_{i}$ at the boundaries. The measure (82) gives a contribution $\int_{0} d \eta \eta^{-2}(\ln \eta)^{-14}$ which is divergent at the lower integration limit and is caused by the $N=0$ state of our general analysis. The $N=1$ state is given by the next order in $\eta$. This 
gives a $\int_{0} d \eta \eta^{-1}(\ln \eta)^{-14}$ which is finite at the lower limit. Note that for the finiteness it was crucial to integrate over the $Y_{1}-Y_{2}$ since this produces the necessary powers of $\ln \eta$. Without the integration the contribution would be $\int d \eta \eta^{-1}(\ln \eta)^{-1}$, which diverges. The term $\epsilon_{-1}$ given by (87) is proportional to $1 / \ln \eta$ and vanishes in the limit $\eta \rightarrow 0$, it can therefor be disregarded in comparison to $\epsilon_{0}$.

For high energy fixed angle scattering the following situation emerges: The power behavior in $s$ is determined by the $\eta$-independent part of $\epsilon_{0}$ and gives the generic behavior $s^{-2}$ which was found away from the boundary of moduli space. The divergence due to the $N=0$ state does not spoil this feature. This analysis does not depend on the specific example chosen as long as one of the $x_{i}$ is finite.

(d) This limit corresponds to the inner radius of the annulus going to zero in the parameterization of (53) this is given by $l \rightarrow \infty$. One can view this as a closed string tadpole were a closed string state propagates along a cylinder of length $l$ and couples to a disk. The divergences come from the closed string tachyon and dilaton at zero momentum propagating for an infinite long time. These are the usual divergences from the closed string sector which are not special to Dirichlet strings. Writing $q=e^{-\pi l}$ the measure (60) gives $\int d q|q|^{-3} \Pi\left(1-|q|^{2 n}\right)^{-24}$ and there are divergent terms coming from $|q|^{-3}$ (tachyon) and $|q|^{-1}$ (dilaton). To consider the limit $l \rightarrow \infty$ the well known representation of the theta function [27] can be used

$$
\theta_{1}(z \mid \tau)=2 q^{\frac{1}{2}} \sum_{n=0}^{\infty}(-1)^{n} q^{n(n+1)} \sin (2 n+1) \pi z
$$

Where $q$ is given by $q=e^{i \pi \tau}$. To lowest order in $\mathrm{q}$ the $\mathrm{q}$ independent term for the Green function $G^{D}(z, w)$ is given by (59)

$$
G^{D}(z, w) \longrightarrow \frac{1}{4 \pi} \ln \left|\frac{\sin \pi(z-w)}{\sin \pi(z-\bar{w})}\right|^{2}+o\left(q^{2}\right)
$$

Mapping the z-plane into the $\rho$-plane via $\rho=\exp (2 \pi i z)$ the cylinder is mapped into the annulus (see figure 5), in this conformal frame the Green function has the form

$$
G^{D}\left(\rho_{z}, \rho_{w}\right) \longrightarrow \frac{1}{4 \pi} \ln \left|\frac{\rho_{z}-\rho_{w}}{1-\rho_{z} \bar{\rho}_{w}}\right|^{2}+o\left(q^{2}\right)
$$


To analyze the fixed angle high energy scattering power behavior in this limit, note that the Green function has a $q$ independent part which in the parameterization of (91) has the form of the Green function for the half plane (48) transformed into the conformal frame of the disk. Hence the properties of the Green function discussed in section 3 are valid. The closed string tachyon and dilaton give divergent contributions, but the fixed angle high energy behavior is governed by the $q$-independent part of (91) and a similar analysis to section 3 and 4 shows that the behavior is still $s^{-2}$ (no vertex operator position has been fixed in contrast to section 3 ). The regularization of the divergence does not spoil this feature.

To summarize we stress that the boundary of moduli space potentially invalidates the analysis of the power behavior of the high energy fixed angle scattering because of the divergences which occur here. For the annulus it has been shown that the divergent contribution and the part responsible for the power behavior can be disentangled and the analysis at generic points of the moduli space is still valid in this limit.

\section{Conclusions}

In this paper we have analyzed the inclusion of Dirichlet boundaries perturbatively in a path integral framework. The inclusion of a finite number of Dirichlet boundaries gives a string theory with novel features and the following points seem to be generic for an any number of boundaries.

High energy fixed angle scattering amplitudes decrease with a power of the center of mass energy. Our analysis of the annulus should carry over to an arbitrary number of boundaries. Generically the Green function vanishes when all vertices are on one boundary and is quadratic in the deviations. The generic behavior for $n$ vertex insertions is then given by $s^{-n / 2}$ from the Gaussian integral over the deviations. In contrast to the analysis of high energy fixed angle scattering for conventional closed strings [23] this behavior comes from a boundary effect and there is no dominating saddle point. This means that there is no simple classical trajectory which dominates the functional integral in a semiclassical approximation. The divergences and the integration over moduli space have to be considered carefully, which makes it difficult to give universal statements for all orders. 
By considering special helicity amplitudes for the scattering of massless tensor states the $N=0$ and $N=1$ divergences have been avoided in the discussion of the fixed angle high energy scattering. The divergences which are analyzed in section 2.3 are a novel feature of Dirichlet strings and there are different ways to make sense out of them. The general rôle of these divergences should be studied further. One way to get rid of the $N=0$ divergence might be to consider a supersymmetric version of a theory with Dirichlet boundary insertions [32].

In general we have to deal with the $N=1$ divergence in the theory. There are two different suggestions of how to deal with them. In one of them [30], the $N=1$ state is interpreted as a Lagrange multiplier field which has to be integrated over (see appendix B), in the other [10], a Fischler-Susskind type of mechanism arises, where the divergences cancel between diagrams of different topology (see appendix $\mathrm{C}$ for a brief description in our context).

Our investigation was limited to a perturbative treatment with a fixed number of boundary insertions. The natural and very difficult question is what happens if one sums over arbitrary many boundary insertions, i.e. generating a condensate of Dirichlet boundaries. It might be possible to make progress by looking at subcritical string theory or matrix models, where one has a handle on nonperturbative questions and one might be able to take the condensation of boundaries into account. Another advantage is that 2-d string theory is a consistent bosonic theory so the divergences coming from the tachyon which are there in critical bosonic string theory are avoided.

Since one motivation for the investigation of Dirichlet string theory is the search for a string theory of QCD, these nonperturbative questions are very important because perturbatively the massless graviton is till present in the spectrum.

\section{Acknowledgements}

I would like to thank M.B. Green for a lot of useful discussions and explanations. I would also like to thank the Theory-Division at CERN for hospitality. This work was supported in part by EPSRC and a Parnett Research Studentship of Churchill College, Cambridge. 


\section{A Modification of the Green function}

This appendix will consider the term $f$ which modifies the Green function $\hat{G}^{D}=G^{D}+f$, from $(24)$

$$
f\left(z_{i}, z_{j}\right)=2 \pi \alpha^{\prime} \sum_{k, l} \oint_{a_{k}} \partial_{w_{1}} G^{D}\left(z_{i}, w_{1}\right) \operatorname{Im} \tau_{k l} \oint_{a_{l}} \partial_{w_{2}} G^{D}\left(z_{j}, w_{2}\right)
$$

With the Green function given by $(\sqrt{16}), \partial_{z} G^{D}(z, w)$ can be expressed in the following way

$\partial_{z} G^{D}(z, w)=-\frac{1}{4} \partial_{z} \ln E(z, w)-\frac{i}{2} \sum_{i, j} \omega_{i}(z) \operatorname{Im} \tau_{i j}^{-1}\left\{\operatorname{Im} \int_{z}^{w} \omega_{j}-\operatorname{Im} \int_{z}^{I(w)} \omega_{j}\right\}$

Note that the line integral can be rewritten into $\int_{w}^{I(w)}$ which is independent of $\mathrm{z}$. The derivative of the prime form $\mathrm{E}$ gives a holomorphic differential of the second kind which vanishes when integrated along a-cycles, using the normalization (6) the line integral around an $a_{l}$-cycle of (93) is given by

$$
\int_{a_{l}} d z \partial_{z} G(z, w)=\frac{i}{2} \sum_{j} \operatorname{Im} \tau_{l j}^{-1} \operatorname{Im} \int_{w}^{I(w)} \omega_{j}
$$

Using this to evaluate (92) gives

$$
f\left(z_{i}, z_{j}\right)=-\frac{1}{4} \sum_{k l} \int_{z_{i}}^{I\left(z_{i}\right)} \omega_{k} I m \tau_{k l}^{-1} \int_{z_{j}}^{I\left(z_{j}\right)} \omega_{l}
$$

We can now reexpress this via the identity

$$
-4 \int_{z}^{I(z)} \omega \int_{w}^{I(w)} \omega=\left(\int_{z}^{I(z)} \omega-\int_{w}^{I(w)} \omega\right)^{2}-\left(\int_{z}^{I(z)} \omega+\int_{w}^{I(w)} \omega\right)^{2}
$$

where the indices of the $\omega_{i}$ are omitted which cannot cause any confusion since the matrix $I m \tau^{-1}$ is symmetric. Now rewrite the line integrals in the following fashion

$$
\begin{aligned}
\left(\int_{z}^{I(z)}-\int_{w}^{I(w)}\right) \omega & =\left(\int_{z}^{I(z)}+\int_{I(z)}^{w}-\int I(z)^{I(w)}\right) \omega \\
& =2 i \operatorname{Im} \int_{z}^{w} \omega
\end{aligned}
$$




$$
\begin{aligned}
\left(\int_{z}^{I(z)}+\int_{w}^{I(w)}\right) \omega & =\left(\int_{z}^{I(z)}+\int_{I(z)}^{I(w)}-\int I(z)^{w}\right) \omega \\
& =2 i \operatorname{Im} \int_{z}^{I(w)} \omega
\end{aligned}
$$

Note that these quantities are independent of the homology, i.e. they are single valued functions on the Riemann surface because the dependence on the homology cancels between the contributions. With the identity above we see that $f\left(z_{i}, z_{j}\right)$ can be cast in the form

$$
\begin{gathered}
f\left(z_{i}, z_{j}\right)=\frac{\pi \alpha^{\prime}}{2} \sum_{k l} \operatorname{Im} \int_{z_{i}}^{z_{j}} \omega_{k} \operatorname{Im} \tau_{k l}^{-1} \operatorname{Im} \int_{z_{i}}^{z_{j}} \\
\quad-\sum_{k l} \operatorname{Im} \int_{z_{i}}^{I\left(z_{j}\right)} \omega_{k} \operatorname{Im} \tau_{k l}^{-1} \operatorname{Im} \int_{z_{i}}^{I\left(z_{j}\right)}
\end{gathered}
$$

This is exactly the quantity needed for the modification in (24).

\section{B The rôle of the $N=1$ state}

This appendix will review the rôle of the level one state in Dirichlet string theory. The factorization on a dividing strip (case b of section 2.3) does not give open string poles in the intermediate states as in the theory with Neumann boundary conditions. It has been suggested in [30] that the $N=1$ state is really a Lagrange multiplier field and that the divergences are caused by the fact that there is no kinetic term for Lagrange multiplier fields and

its propagator becomes singular. The issue concerns the quantization of a free open string on an infinite strip (as in (27)) with boundary condition $Y_{1}=Y_{2}$ - both boundaries are at the same point in space time. The zero mode of the energy momentum tensor is then independent from the position of the boundary $Y: L_{0}=N$. The physical state condition on a state $|\Phi\rangle$ is $\left(L_{0}-1\right)|\Phi\rangle=0$ and is trivially satisfied for $|\Phi\rangle=\omega(Y)_{\mu} a_{-1}^{\mu}|o\rangle$. So there is no condition on the wave function $\omega(y)_{\mu}$. This becomes clearer if the reparameterization ghosts are included and the BRST-cohomology of free string fields is used. The BRST-operator [31] for Dirichlet strings is given by

$$
Q_{B R S T}=: \sum_{m} c_{m}\left\{L_{-m}^{X}+\frac{1}{2} L_{-m}^{g h}-1 \delta_{m, 0}\right\}
$$


Where $L_{m}^{X}$ is given by the modes of the $X$-part of the energy momentum tensor and $L_{m}^{g h}$ are the usual modes of the bc-ghosts energy-momentum tensor. Since the boundary conditions of the space time fields do not influence the local geometry on the world sheet, the bc-ghost do not feel the Dirichlet boundary conditions. The most general field of ghost number $-\frac{1}{2}$ at level 1 is given by the following field

$$
\left(\omega(Y)_{\mu} a_{-1}^{\mu}+\lambda(Y) c_{0} b_{-1}\right)|\downarrow\rangle
$$

(following the conventions of [31]). The state $|\downarrow\rangle$ is the state annihilated by all positive ghost modes and has ghost number $-\frac{1}{2}$. For free string-fields $|\Phi\rangle$ the condition $Q_{B R S T}|\Phi\rangle=0$ generates the linearized equations of motion for the wavefunctions $\omega(Y)$ and $\lambda(Y)$. Gauge transformations on the Level one fields $\delta|\Phi\rangle=Q_{B R S T}|\Psi\rangle$ are given by the general level one field of ghost number $-3 / 2:|\Psi\rangle=\rho(Y) b_{-1}|\downarrow\rangle$ (In the Neumann case this gives The Maxwell equation for $\omega$ if the auxiliary field $\lambda$ is integrated out). Here the situation is entirely different, there are no conditions on the wave-function at all. This seems to be an indication that the level one open string field is a Lagrange multiplier field and that the proper way to deal with it is to

integrate it out. This is very difficult since as we have seen this state couples to closed string (scalar) states and it influences the closed string spectrum. An investigation of this question is given in [30] where it is indicated that the dilaton might be removed from the theory.

\section{Cancellation of infinities}

As mentioned in section 2 Polchinski has introduced a different version of Dirichlet string theory. In this scheme all boundaries are mapped to the same space time point. In the calculation of scattering amplitudes one usually takes just connected diagrams into account (the part of the S-matrix which does not have trivial delta functions in a subset of momenta is important). In this scheme the disconnected world sheet can exchange momenta via their common space time point and so they are not disconnected from a space time point of view. The power of the closed string coupling constant $g_{c l}$ is given in terms of the number of diagrams $m$, the number of vertices $k_{i}$, the number of boundaries $b_{i}$, the number of handles $h_{i}$ on diagram $i$, where $i=1, . ., m$

$$
g_{c l}^{\left\{-2 m+\sum b_{i}+\sum k_{i}+2 \sum h_{i}\right\}}
$$


This changes in the way the perturbation expansion is organized has two notable consequences, namely that for every diagram an arbitrary number of disks with no vertex operator insertions has to be introduced each of which gives a contribution $\left(C g_{c l}^{-1}\right)^{n} / n$ ! for $n$ disks. Summing over $n$ gives a factor $\exp \left(C / g_{c l}\right)$ where $C$ is a negative constant. The high energy fixed angle behavior is again power behaved but differs from that in our approach since there are configurations where every vertex operator is on a separate disk, which gives a momentum independent $s^{0}$ contribution. Polchinski has shown that for the $N=1$ divergences which we analyzed in Section 2.3 there is a cancellation between the cases (a), (b) and (c) if one considers diagrams of the same order in $g_{c l}$ but of different topology. Note that the $N=1$ state which is responsible for the divergence enters the amplitudes in the following way

$$
\left\langle V_{1} . . V_{k} \oint \partial_{n} X_{\mu}\right\rangle_{\Sigma}=\sum_{j=1}^{k} k_{j}^{\mu}\left\langle V_{1} . . V_{k}\right\rangle_{\Sigma}
$$

Here $V_{i}$ denotes a physical state vertex operator with momentum $k_{i}^{\mu}$ and $\langle. .\rangle_{\Sigma}$ denotes the functional integral over the moduli space of $\Sigma$. As an example we now indicate how the divergences in Figure 3 cancel in this scheme. The $N=1$ divergent contribution of the diagram (3.a) is given by

$$
\begin{gathered}
A_{(a)}=\int \frac{d \eta}{\eta}\left\langle V_{1} V_{2} V_{3} \oint \partial_{n} X^{\mu}\right\rangle_{\Sigma}\left\langle\oint \partial_{n} X_{\mu} V_{4}\right\rangle_{D} \\
=\int \frac{d \eta}{\eta}\left\langle V_{1} V_{2} V_{3}\right\rangle_{\Sigma}\left\langle V_{4}\right\rangle_{D}\left(k_{1}+k_{2}+k_{3}\right)_{\mu} k_{4}^{\mu}
\end{gathered}
$$

To cancel this divergence we add two more diagrams which contain divergences of type (c) in order to give an complete square of momenta $\left(\sum_{i=1}^{4} k_{i}\right)^{2}$ which vanishes upon momentum conservation, since the single boundary position $Y$ is integrated. The two diagrams are given by

$$
\begin{aligned}
A_{(a)}^{\prime} & =\frac{1}{2} \int \frac{d \eta}{\eta}\left\langle V_{1} V_{2} V_{3} \oint \partial_{n} X^{\mu} \oint \partial_{n} X_{\mu}\right\rangle_{\Sigma}\left\langle V_{4}\right\rangle_{D} \\
& +\frac{1}{2} \int \frac{d \eta}{\eta}\left\langle V_{1} V_{2} V_{3}\right\rangle_{\Sigma}\left\langle\oint \partial_{n} X^{\mu} \oint \partial_{n} X_{\mu} V_{4}\right\rangle_{D} \\
& =\frac{1}{2} \int \frac{d \eta}{\eta}\left\langle V_{1} V_{2} V_{3}\right\rangle_{\Sigma}\left\langle V_{4}\right\rangle_{D}\left\{k_{4}^{2}+\left(k_{1}+k_{2}+k_{3}\right)^{2}\right\}
\end{aligned}
$$


$A_{(a)}+A_{(a)}^{\prime}$ vanishes now because of momentum conservation. The factor $1 / 2$ comes from the symmetry factor for the second diagrams. The divergence of diagram (3.b) is cancelled in a similar way

$$
\begin{aligned}
A_{(b)} & =\int \frac{d \eta}{\eta}\left\langle V_{1} V_{2} \oint \partial_{n} X^{\mu}\right\rangle_{\Sigma_{1}}\left\langle\oint \partial_{n} X_{\mu} V_{3} V_{4}\right\rangle_{\Sigma_{2}} \\
A_{(b)}^{\prime} & =\int \frac{d \eta}{\eta}\left\langle V_{1} V_{2} \oint \partial_{n} X_{\mu} \oint \partial_{n} X^{\mu}\right\rangle_{\Sigma_{1}}\left\langle V_{3} V_{4}\right\rangle_{\Sigma_{2}} \\
& +\int \frac{d \eta}{\eta}\left\langle V_{1} V_{2}\right\rangle_{\Sigma_{1}}\left\langle\oint \partial_{n} X_{\mu} \oint \partial_{n} X^{\mu} V_{3} V_{4}\right\rangle_{\Sigma_{2}}
\end{aligned}
$$

Note that in this cancellations it is actually not enough to just look at planar diagrams as an example, the amplitudes $A_{(a)}^{\prime}$ and $A_{(b)}^{\prime}$ contain the insertion of the two boundary vertex operators at different components of the boundary and this corresponds to a degeneration of a nonplanar surface. The divergence of type (c) in the case of a single surface is actually zero, since

$$
\begin{gathered}
A_{(c)}=\int \frac{d \eta}{\eta}\left\langle V_{1} V_{2} V_{3} V_{4} \oint \partial_{n} X^{\mu} \oint \partial_{n} X_{\mu} V_{4}\right\rangle_{\Sigma} \\
=\int \frac{d \eta}{\eta}\left\langle V_{1} V_{2} V_{3} V_{4}\right\rangle_{\Sigma}\left(k_{1}+k_{2}+k_{3}+k_{4}\right)^{2}
\end{gathered}
$$

It is clear that this method can be applied to the divergences (a) to (c) in general. This indicates that the divergence problem of the insertion of Dirichlet boundaries might not make the theory inconsistent but lead to some new interesting physics.

\section{References}

[1] J.L. Cardy, Nucl. Phys B324 (1989) 581

[2] J. Schwarz, Nucl. Phys. B65 (1973) 131

[3] E. Corrigan and D. Fairlie, Nucl. Phys. B91 (1975) 527

[4] M.B. Green J.A. Shapiro, Phys. Lett. B64 (1976) 454

[5] A.Cohen et al., Nucl. Phys B281 (1987) 127 
[6] M.B. Green, Phys. Lett. B266 (1991) 325

[7] J. Dai,R.G. Leigh and J. Polchinski, Mod. Phys. Lett. A4 (1989) 2073

[8] M. Li, Nucl. phys B420 (1994) 339

[9] Z.Yang preprint UR-1288/ER-40685-727 (unpublished)

[10] J.Polchinski, Phys. Rev D50 (1994) 6041

[11] A.A. Belavin and V.G. Knizhnik, Phys. Lett. B168 (1986) 201

[12] E. Verlinde and H. Verlinde, Nucl. Phys. B288 (1987) 357

[13] M. Shiffer and D.C. Spencer, Functionals of finite Riemann surfaces (Princeton Univ. Press, 1954)

[14] C.P. Burgess and T.R. Morris, Nucl. Phys B291 (1987) 256

[15] S.K. Blau et. al., Nucl. Phys. B301 (1988) 285

[16] M. Bianchi and A.Sagnotti, Phys. Lett. B231 (1989) 389

[17] E'Dhoker and D.H. Phong, Rev. of mod. Phys. 60 (1988) 917 and references therein

[18] M.B. Green, Nucl. Phys. B124 (1977) 461

[19] M.B. Green, Phys. Lett. B282 (1992) 380

[20] J.Polchinski, Phys. Rev. Lett. 68 (1992) 1267

[21] G. Veneziano, Nuov. Cim. 57a (1968) 190

[22] V. Allesandrini,D. Amati and B. Morel, Nuov. Cim. 7A (1971) 797

[23] D.J. Gross and P.F. Mende, Nucl. Phys. B303 (1988) 407

[24] C.G. Callan et al.,Nucl. Phys. B293 (1987) 83

[25] H. Farkas and I. Kra, Riemann surfaces (Springer, Berlin, 1980)

[26] J. Fay, Theta functions on Riemann Surfaces, Lect. notes in math. 323 (Springer, Berlin 1973) 
[27] Erdelyi et al., Bateman manuscript (McGraw-Hill, New York, 1955)

[28] N. Marcus, Phys. Lett. B219 (1989) 265

[29] W. Fischler and L. Susskind, Phys. Lett. B171 (1986) 383

[30] M.B. Green, Phys. Lett. B302 (1993) 29

[31] M.B. Green,J.H. Schwarz and E. Witten, Superstring theory (Cambridge University Press,1987)

[32] M.B. Green, Phys. Lett. B329 (1994) 435

[33] E. Gava et al., Phys. Lett. B168 (1986) 207

[34] J. Polchinski, Nucl. Phys. B307 (1988) 61

[35] H. Sonoda, Nucl. Phys. B311 (1989) 401,417 


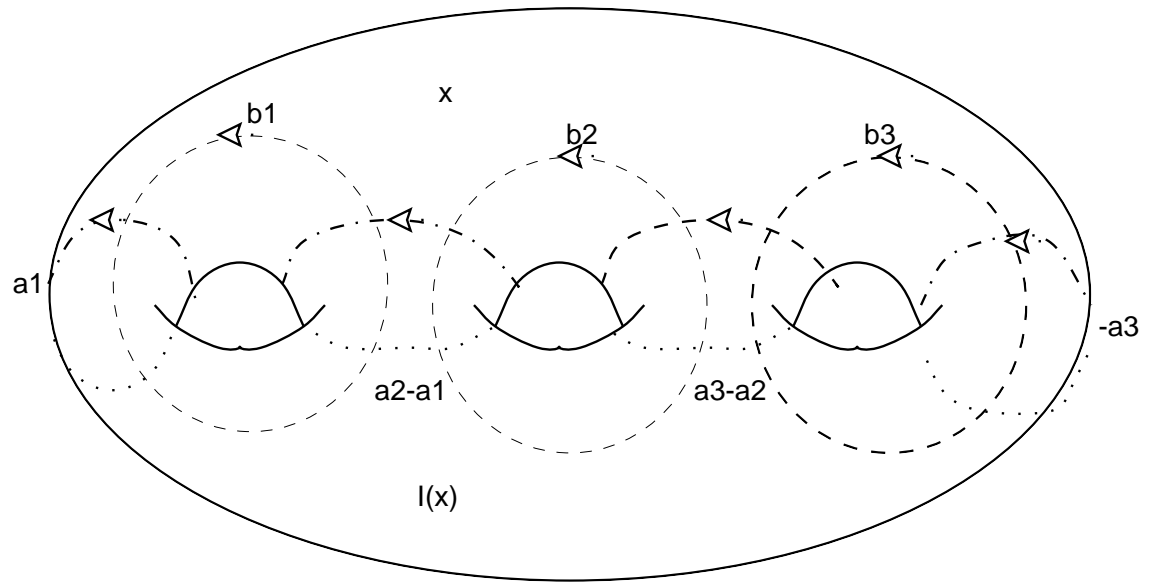

Figure 1: Canonical homology cycles on a surface of genus 3, The involution I acts in a way that the linear combinations of a cycles are fixed under I 


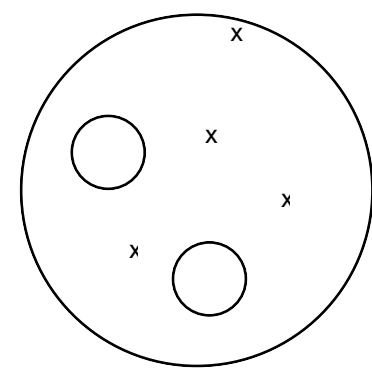

(a)

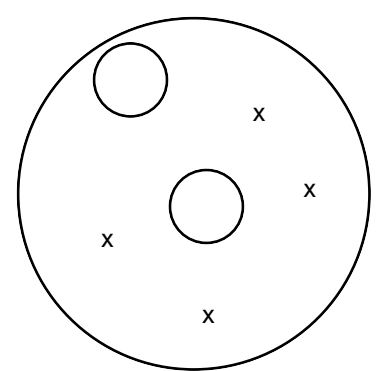

(c)

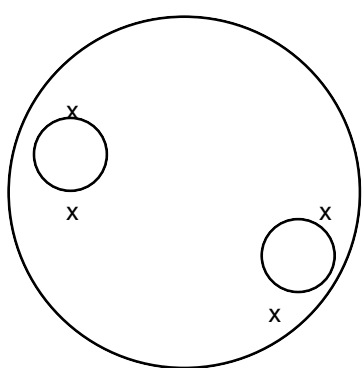

(b)

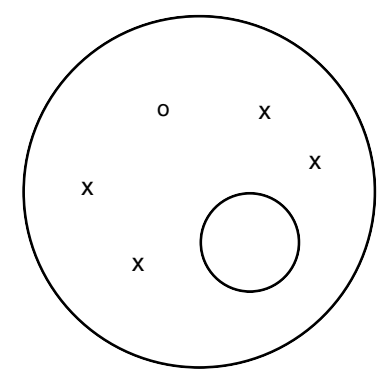

(d)

Figure 2: The degenerations of an open string with three boundaries and for vertex operators: a) one vertex going to the boundary b) surface is separated into two parts c) two boundaries touching d) one boundary shrinking to a point 


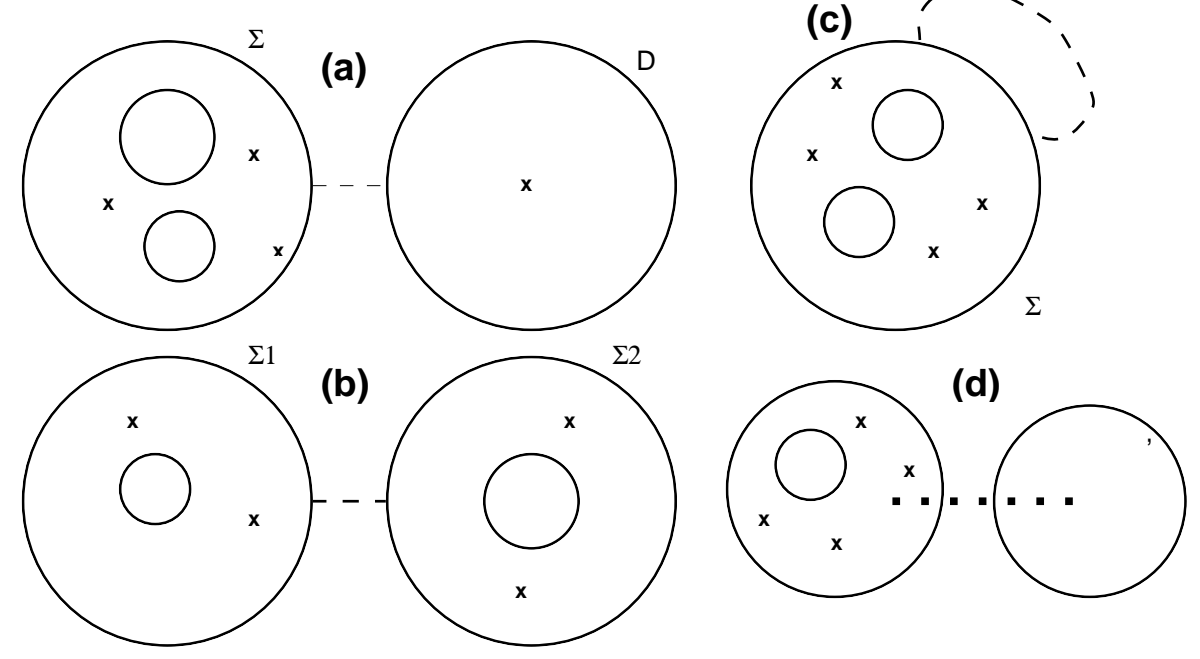

Figure 3: a)-c) The degenerations represented by the insertion of two boundary vertex operators and a open string propagator d) the degeneration is represented by closed string vertex operator on the surface and a closed string propagator coupling to a disk 


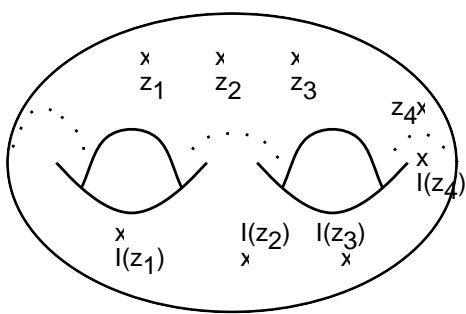

(a)

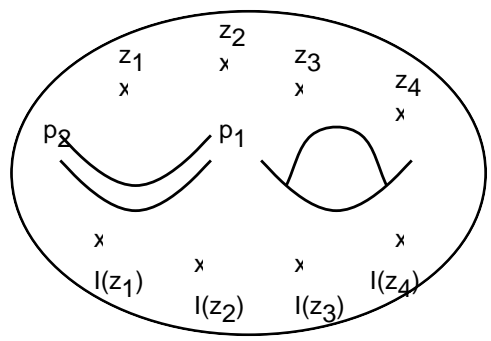

(c)
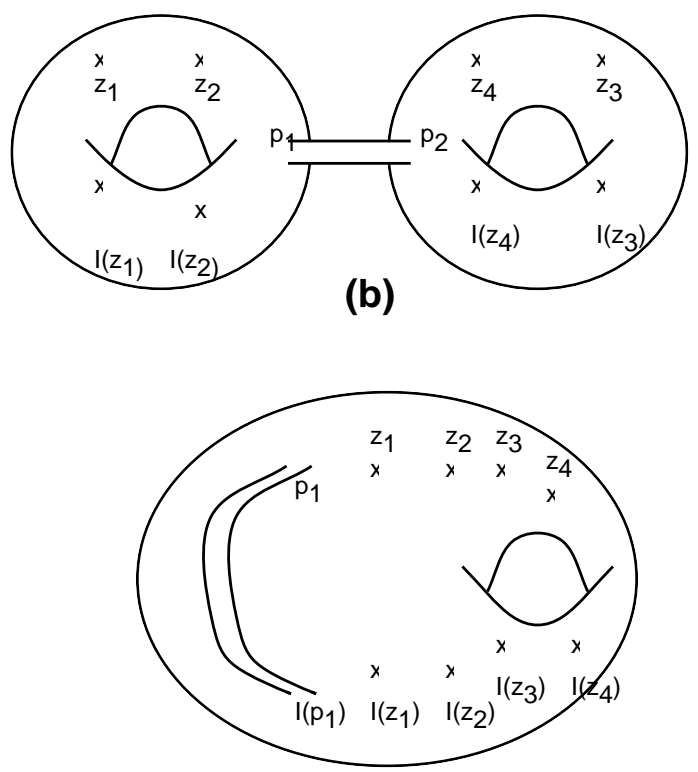

(d)

Figure 4: The degenerations of open strings represented as degenerations of the doubled surface which is symmetric under I a) one vertex and its mirror image coming close at the boundary b) a zero homology cycle pinching separating the surface into disconnected parts c) a b-cycle pinching, for b), c) the plumbing fixtures $p_{1}$ and $p_{2}$ are invariant under I, d) an a-cycle pinching 


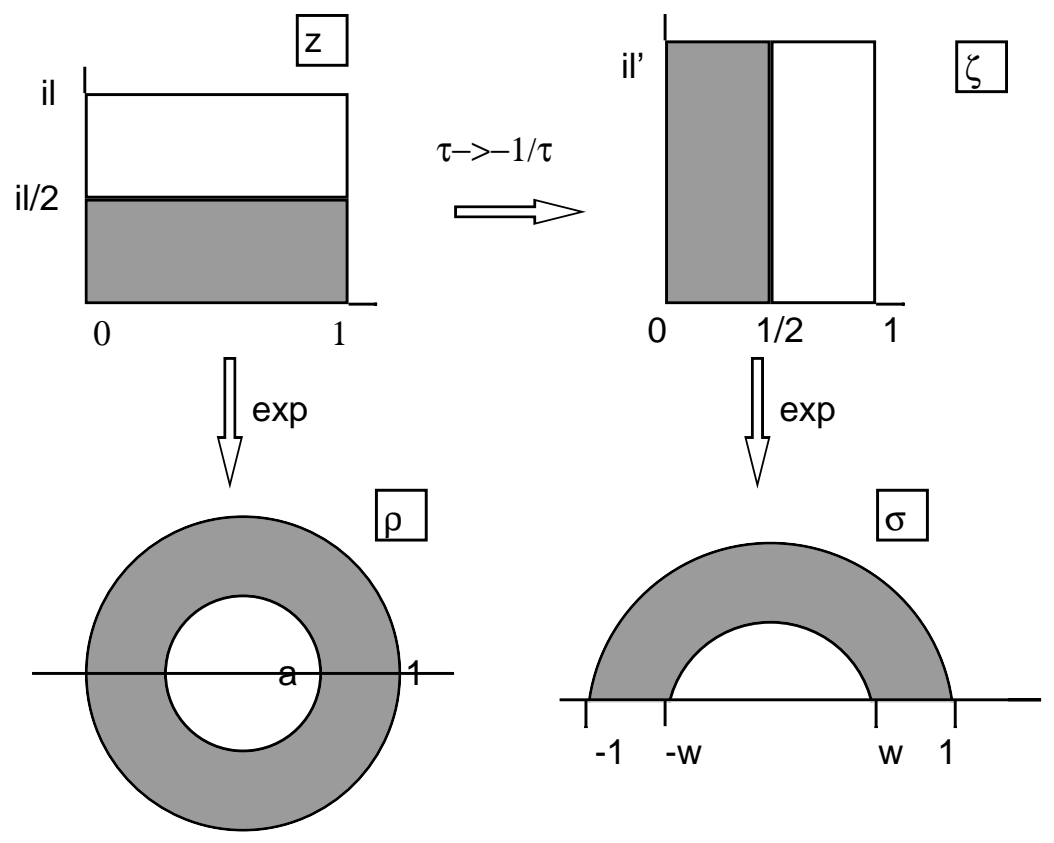

Figure 5: The different variables used for the annular world sheet. The $z$ plane represents the fundamental cell, the $\zeta$ plane the fundamental cell after the modular transformation. The annulus in the $\rho$ plane and the semiannulus in the $\sigma$ plane are given by exponential mappings. 